\title{
Virulence shift in a sexual clade of Type X Toxoplasma infecting Southern Sea Otters
}

\author{
KENNARD, $\mathrm{A}^{1}$, MILLER, MA ${ }^{2}$, KHAN, $\mathrm{A}^{1^{*}}$, QUINONES, $\mathrm{M}^{3}$, MILLER, $\mathrm{N}^{4}$, SUNDAR, ${ }^{1}, \mathrm{JAMES}$, \\ $\mathrm{ER}^{5}$, ROOS, DR ${ }^{4}$, CONRAD, $\mathrm{PA}^{6}$ AND GRIGG, $\mathrm{ME}^{1,5}$. \\ ${ }^{1}$ Laboratory of Parasitic Diseases, NIAID, National Institutes of Health, Bethesda, MD, USA. ${ }^{2}$ California \\ Department of Fish and Game, Santa Cruz, CA, USA. ${ }^{3}$ Bioinformatics and Computational Biosciences Branch, \\ NIAID, National Institutes of Health, Bethesda, MD, USA. ${ }^{4}$ Department of Biology and Penn Genome Frontiers \\ Institute, University of Pennsylvania, Philadelphia, Pennsylvania, USA. ${ }^{5}$ Department of Medicine, University of \\ British Columbia, Vancouver, BC, Canada. ${ }^{6}$ School of Veterinary Medicine, University of California, Davis, CA, \\ USA. \\ ${ }^{*}$ Current Address: Agricultural Research Service, USDA, Beltsville, MD, USA.
}

\begin{abstract}
How virulent protozoal pathogens capable of causing overt disease are maintained in nature is an important paradigm of eukaryotic pathogenesis. Here we used population genetics and molecular methods to study the evolution and emergence of a marine invasion of new genetic variants of Toxoplasma gondii, referred collectively as Type X (HG12). 53 Toxoplasma isolates were obtained from mustelids that stranded between 1998-2004 with toxoplasmosis (ranging from chronic infection to fatal encephalitis). Over $74 \%$ of the sea otters collected throughout their geographic range were infected with Type X as determined by multi-locus PCR-DNA sequencing. Depending on the locus investigated, Type X strains possessed one of three allelic types that had independently assorted across the strains examined; either genetically distinct alleles, referred to as " $\gamma$ " or " $\delta$ ", or a Type II allele. Phylogenetic incongruence among locus-specific trees, genome-wide CGH array and WGS analyses confirmed that Type $\mathrm{X}$ is a sexual clade of natural recombinants that resemble $\mathrm{F} 1$ progeny from a genetic cross between Type II and a mosaic of two distinct " $\gamma$ " or " $\delta$ " ancestries. A single Type X genotype $(19 / 53 ; 36 \%)$ had expanded in sea otters largely as subclinical chronic infections, but it was highly pathogenic to mice $\left(\mathrm{LD}_{100}=1\right.$ parasite). To determine whether murine virulence genes could be mapped within this naturally occurring population, we performed a genome scan and identified four QTLs with LOD scores greater than 4.0. Targeted disruption of ROP33, the strongest candidate from among 16 genes within the highest QTL on Chromosome VIIa established ROP33 as a murine virulence locus. The ability of this highly pathogenic clone to expand and cause the majority of sea otter infections supports a virulence shift model whereby generalist pathogens like Toxoplasma utilize their sexual cycles to produce new strains with an expanded biological potential. Such a trait enables pathogens to extend their host range or be naturally selected within their vast intermediate host range to maximize their transmission. Our work thus establishes a rationale for how virulent strains can be maintained cryptically in nature across a pathogen's broad host range, and act as reservoirs for epidemic disease.
\end{abstract}

\section{Importance}

43 Waterborne outbreaks of protozoal parasites are increasingly causing fatal disease in a wide range of animals, including humans. Population expansion of felids near marine estuarine environments has led to increased exposure of marine wildlife to highly infectious Toxoplasma gondii oocysts shed in the feces of cats that are dispersed by storm events. In 47 North America Toxoplasma is thought to possess a highly clonal population structure 
dominated by 4 clonal lineages (I, II, III, and X). Population genetic analysis of 53 Toxoplasma isolates collected longitudinally from mustelids infected with Toxoplasma that stranded between 1998-2004 identified a majority of otters (74\%) to be infected with Type X Toxoplasma, and that Type $\mathrm{X}$ is not a clonal lineage, but rather a recombinant clade of strains consistent with a recent genetic cross that produced at least 12 distinct haplotypes. Importantly, one Type $\mathrm{X}$ haplotype expanded in $36 \%$ of otters across their geographic range and caused relatively benign infections, however it was highly pathogenic to mice. A genome scan was performed to identify a new virulence locus, a secreted serine threonine kinase (ROP33), that is pathogenic in mice, but not sea otters. Our data support a virulence shift model whereby generalist pathogens like Toxoplasma utilize their sexual cycles to produce virulent strains that are maintained cryptically in nature, according to their differential capacity to cause disease within the pathogen's broad intermediate host range. This type of "zoonotic selection" has important public health implications. Strains capable of causing fatal infections can persist in nature by circulating as chronic infections in intermediate host species that act as reservoirs for epidemic disease.

\section{Introduction}

How successful microbes maintain reservoirs of virulent strains in nature is understudied and is an important paradigm of infectious diseases. Whereas many studies document how microbial pathogens acquire or admix genetic material to influence their pathogenicity, relatively less is known how emergent strains are selectively maintained in nature. Specifically, it is well established that viruses utilize reassortment to rapidly produce admixture lines that possess a range of altered biological potential, including virulence [1]. Bacteria transfer mobile plasmid elements that encode pathogenicity islands via conjugation to confer an altered virulence potential on those strains that receive them [2-4]. Among both fungi and protozoa, genetic hybridization by sexual recombination is a potent way to produce genomic variation or new admixture lines that possess increased virulence and are capable of causing disease outbreaks or altering host range [5-7]. What is less clear are the mechanisms at play allowing highly prevalent pathogens to maintain virulent strains cryptically in nature. We sought to test whether generalist pathogens, such as the protozoan parasite Toxoplasma gondii, recovered from natural infections in one animal host, leverages its broad range of intermediate hosts to selectively partition parasite genotypic diversity and virulence potential.

Toxoplasma gondii is a highly successful and prevalent protozoan pathogen that infects a wide range of wildlife, livestock and $20-80 \%$ of humans worldwide [8-10]. Its success in nature is largely attributed to its highly flexible life cycle. It is propagated asexually by carnivory among essentially all warm-blooded vertebrates (via orally infectious tissue cysts) or sexually within its definitive felid host by self-mating (when a single parasite undergoes fertilization and sexually clones itself) or by out-crossing (producing as many as $10^{8}$ genetic hybrids that are transmissible as environmentally stable, highly infectious oocysts). Genetic hybridization has been previously shown to produce genotypes that possess a wide spectrum of biological potential, including altered virulence or a capacity to expand as successful epidemic clones [7, 11, 12]. Importantly, parasite clones possess a broad spectrum of disease states that are highly dependent on both parasite genetics and the animal species infected [8, 13-15]. In rodents, low dose inoculums of Type I Toxoplasma

93 strains are uniformly lethal to laboratory mice [16] whereas Type II strains are considerably 
94 less virulent and routinely establish chronic, transmissible infections [14]. In contrast, wild95 derived CIM mice that encode resistant alleles of immunity-related GTPases (IRGs) confer 96 resistance to virulent Type I strains by inactivating parasite-specific secreted kinases 97 (ROP18/ROP5) that control mouse susceptibility to infection $[17,18]$. The end result of this 98 host genetic, parasite genetic interplay determines the potential for a disease-producing 99 virulent clone to expand or be maintained cryptically within a strain-specific, or host species100 specific, manner, but this has not so far been demonstrated to occur in nature and has only been inferred by laboratory studies. Likewise, laboratory rats are resistant to Type I infections and fail to transmit parasites, but again this is entirely rat species-dependent [19]. The molecular basis for this resistance is an allele-dependent activation of the NLRP1 inflammasome resulting in macrophage pyroptosis and the inhibition of parasite growth in Lewis (LEW) and Sprague Dawley (SD) rats, but not in Brown Norway (BN) or Fischer CDF rats, which go on to establish chronic, transmissible infections of mouse-virulent Type I strains [20-22]. While these laboratory studies suggest the possibility that virulent strains can be maintained cryptically across the pathogen's host range [23], the phenomenon has not been systematically investigated nor demonstrated to occur in a natural setting.

Between 1998 and 2004, Toxoplasma gondii emerged as a significant disease in California sea otters (Enhydra lutris nereis), a federally listed threatened species [24, 25]. Full necropsies performed on each stranded sea otter identified a wide spectrum of disease sequelae among Toxoplasma-infected otters, ranging from chronic asymptomatic infection to fatal meningoencephalitis at time of death $[25,26]$. Importantly, the majority of sea otters were found to be infected by a single outbreak clone, referred to as Type X (or HG12) [24]. Based on multi-locus PCR-DNA sequencing (MLST) using a limited set of genotyping markers, Type X was defined as the fourth clonal lineage in the US [27] and it is highly prevalent in sylvatic niches as chronic, subclinical or mild infections in $47 \%$ of US wildlife $[28,29]$. The range of disease sequelae detected in sea otters, however, was not consistent with infection by a single clone. Other possibilities proposed to explain variation in disease susceptibility have included co-infection [30], exposure to environmental pollutants, or toxins that cause immunosuppression [31]. More recently, WGS performed on Toxoplasma isolates has called into question whether MLST analyses are sufficiently resolved to predict clonotypes because they fail to capture extant genetic heterogeneity [15]. Indeed, a previous study concluded that Type X was comprised of two genotypes [32], but these two genotypes differed by only a few SNPs, so it was not clear whether strain variation was likewise playing a contributing role in sea otter disease in this novel host-parasite interaction.

To test for this and to determine the true extent of genetic diversity among naturally circulating Type $\mathrm{X}$ isolates, we sequenced a wide selection of linked and unlinked markers across the nuclear and organellar genomes for 53 isolates collected longitudinally from infected sea otters across a seven year period. Based on these results, we selected 16 isolates for WGS. Analysis of our data support a model whereby Type X exists as a recombinant clade of strains that resemble F1 progeny from a natural cross between a Type II strain and a novel genotype that has not been identified previously in nature. We show that one sea otter genotype is widely distributed and has expanded to cause the majority of infections, which were largely subclinical and consistent with a strain that has been naturally selected in sea otters. This genotype, however, was highly pathogenic to laboratory mice and a population level, forward genetic approach using WGS mapped a mouse virulence gene to a novel parasite-specific secreted kinase ROP33, also referred to as WNG3 [33]. Our natural 
140 population dataset argues that Toxoplasma leverages its broad range of intermediate hosts 141 to partition genetic diversity. Further, that intermediate hosts play a central role in the 142 natural selection, expansion and maintenance of cryptically virulent strains in a host speciesspecific manner and that these infected hosts act as reservoirs for pathogenic or epidemic disease in another host species.

\section{Results}

\section{Genetic characterization of Toxoplasma gondii strains isolated from sea otters}

In the late 1990s, an increasing number of sea otters stranded along the California coast with protozoal encephalitis due to Toxoplasma gondii [25, 29, 32, 34]. An initial genetic analysis performed on a subset of $35 \mathrm{~T}$. gondii isolates established that 21 were a new genetic type, referred to as Type X (also known as Haplogroup 12, or HG12), but this conclusion was limited to sequence typing at a single locus, GRA6 [25]. We developed a multi-locus (MLST) PCR-DNA sequence-based typing scheme using an additional 4 unlinked markers (BSR4, BAG1, SAG3, and ROP1) that possess a wide-range of phylogenetic strength, including markers under neutral (BSR4), diversifying (GRA6, SAG3, ROP1), and purifying (BAG1) selection to determine the molecular genotypes of $53 \mathrm{~T}$. gondii isolates collected longitudinally over a seven-year period between 1998-2004 from fresh, beachcast California sea otters. Isolates were obtained throughout the California sea otter range (Supplemental Figure 1). Antibody titers against Toxoplasma (when available), stranding location, otter sex, and co-infection status with other protozoal agents was available for 52 of the sea otters (Supplemental Figure 1).

\section{Point-source outbreak of Type II strains infecting sea otters}

To determine the T. gondii genotypes among the sea otter isolates, the 5 locus MLST was applied. Typing at just the GRA6 marker identified three sequence types, either a Type II allele in 16 (30\%) isolates, or two non-archetypal alleles, previously identified as X or A $[25,32]$, in $25(47 \%)$ and $12(23 \%)$ isolates, respectively. Expanding the genetic analysis across five markers showed that 2 of 16 isolates with a Type II allele at GRA6 were predicted to be non Type II, recombinant strains, because they possessed non-archetypal alleles at ROP1 (3675) or BSR4 and BAG1 (3671) (Figure 1A). Of the remaining 14 otters infected with Type II strains, 12 (86\%) were identified within a restricted geographic range, a 40 mile stretch off the California coast line containing Moss Landing and Monterey Bay, which is consistent with a point source outbreak of a Toxoplasma strain that is found most frequently infecting human and domestic livestock in the USA. The other two Type II infected otters were recovered from Pismo Beach in Central California. Further, the majority of Type II infected otters $(9 / 14 ; 64 \%)$ were collected during the first half of the study (Supplemental Figure 1). Males were predominantly infected with this genotype, and it was the only genotype that had a male-specific infection sex bias. [Fisher's two-sided exact test II vs. all; $\mathrm{p}=0.008]$

\section{Type $X$ is a recombinant clade of strains}

Of the remaining 39 (74\%) Toxoplasma isolates, collectively referred to as Type X or A by the allele present at GRA6, a maximum of only three alleles were identified at the additional sequenced loci, either a canonical Type II allele or one of two genetically distinct alleles, 
186 referred to as " $\gamma$ " or " $\delta$ ", that appeared to segregate independently across the isolates (Figure 1A). In total 8 distinct haplotypes designated A-H were resolved based on their differential inheritance of the limited alleles, consistent with recombination between a Type II strain and a mosaic of two distinct ancestries as the most plausible explanation for the genetic relationship among the non-Type II strains. The Type $\mathrm{X}$ haplotype designated A was dominant $(19 / 39 ; 49 \%)$. It was widely distributed across the entire geographic range of the Southern sea otter (Supplemental Figure 1), and it was isolated in every year except 2000. Importantly, this genotype had expanded during the time frame of the study period, and it was responsible for the majority of infections by 2003 (Figure 1B).

\section{Acute virulence in mice is dependent on Type $X$ haplotype} characterize the otter isolates, 18 strains, including two Type II and at least one isolate representing each of the 8 distinct Type $\mathrm{X}$ haplotypes, were tested for their mouse virulence phenotype. Isolates were selected based largely on their ability to expand in vitro in human foreskin fibroblasts. Virulence was assayed in female CD-1 outbred mice using a low-dose, 50 tachyzoite, intra-peritoneal injection model. At this inoculum, mouse avirulent strains (e.g. Type II) establish chronic, transmissible infections whereas mouse virulent strains (e.g. Type I) die acutely within 10-14 days.

Among the otter isolates, three mouse virulence phenotypes were identified: virulent (red; all mice died acutely), intermediate virulent (blue; some mice survived acute disease), and avirulent (green; all mice survived acute infection and produced chronic disease). The two otter isolates that possessed a Type II MLST were avirulent (Figure 1C; green) and phenocopied the infection kinetics of two well studied Type II lines Me49 and 76K. Among the Type X haplotypes, E and G were also avirulent. Three otter isolates from haplotype A, the genotype that had expanded to cause the majority of sea otter infections, were highly pathogenic to mice; all infected mice died acutely (Figure 1C; red). Haplotypes D and H were likewise highly virulent in mice. The three haplotype $\mathrm{F}$ otter isolates all possessed an intermediate virulence phenotype (Figure 1C; blue). Of particular note, the 5 isolates from haplotypes $\mathrm{B}$ and $\mathrm{C}$ had a mix of two mouse virulence phenotypes, they were either avirulent (green) or they were intermediate virulent (blue) (Figure 1C). Because the Type X isolates displayed a range of virulence phenotypes, the data collectively support a model whereby Type X represents a clade of recombinants from a genetic admixture of a mouse avirulent Type II strain with at least one other parent that is mouse virulent, rather than expansion of a single clonal lineage. Furthermore, not all isolates within each subgroup displayed equivalent virulence kinetics, in particular isolates from the $\mathrm{B}$ and $\mathrm{C}$ haplotypes were either avirulent or possessed intermediate virulence, suggesting that the 5 marker MLST was insufficiently resolved to fully capture total genetic and phenotypic diversity among the isolates recovered from infected Southern sea otters.

To explore this possibility, the 18 isolates assayed through mice plus 3 additional isolates, one each from haplotypes A, C and D, were further genotyped at 18 markers (17 nuclear-encoded single copy gene loci plus one microsatellite marker) representing an expanded set of linked and unlinked loci across the genome. Additionally, 2 markers, one each from the organellar genomes of the apicoplast and mitochondria, were included to determine the ancestry of each maternally inherited genome. This represents a significant increase in the number of markers that were previously used to conclude that Type X (HG12) 
is a clonal lineage [10, 27, 36]. Altogether, the 20 marker MLST method provided 15,404 bp of sequence information to determine the genetic relationship among Toxoplasma strains isolated from Southern sea otters (Supplemental Table 1).

\section{Type $X$ is comprised of 12 distinct haplotypes by an expanded MLST analysis}

To establish the number of haplotypes among the 21 isolates, sequence data for the 17 nuclear-encoded single copy gene loci was concatenated and analysed using eBURST (Figure 1D). eBURST resolves strains into clonal complexes (CC) that are in linkage disequilibrium. In this case, coded MLST data was used to identify strains that differ at one locus (or share 16 out of 17 markers) and isolates that differed at the same genetic locus were grouped together and connected by a line within each clonal complex. As expected, eBURST clustered the two Type II isolates $(2987,3131)$ into a single clonal complex with Me49 (CC1). Type X subgroup G isolate 3675, originally identified by its allele at the microsatellite marker ROP1, was included within CC1 because it differed from Type II strains at only SAG4. For the remaining 18 Type X isolates, 15 clustered into 3 clonal complexes (designated CC2-CC4), and 3 unique haplotypes were resolved. The eBURST analysis failed to support a clonal lineage designation for Type X. Further, the increased resolution provided by the 17 markers subdivided the 8 distinct haplotypes defined using 5 loci in Figure 1A into 12 haplotypes (Figure 1D) which was consistent with another study that resolved Type X Toxoplasma genotypes isolated from southern sea otters into 8 genotypes [26]. One isolate each from subgroups $A(n=4 ; 3168), C(n=4 ; 3160), D(n=3 ; 4166)$ and $F(n=3 ; 3387)$ was resolved further as a unique haplotype based on their inheritance of alleles at PK1, SAG4 and GRA7 (Supplemental Table 1).

\section{Type $X$ resembles a recombinant clade of $F 1$ progeny from a natural cross}

eBURST is insufficiently resolved to distinguish among isolates that exist either as variants within a clonal lineage, because they possess alleles that differ by only minor mutational drift, or as genetic admixtures, that possess genetically distinct alleles derived from different parental types. Previous studies suggested that Type X (or HG12) is a clonal lineage infecting sea otters and wildlife in North America, and that HG12 represents an expanded clone from a sexual cross between a Type II strain and a new genetic lineage, that was referred to as " $\gamma$ ", but this was based largely on the alleles found at just two loci, GRA6 and GRA7 [24, 25, 27]. To investigate whether the genetic relationship among the 12 distinct haplotypes is supported by a model of genetic drift, or that of recombination, individual maximum likelihood trees were created for each of the 17 nuclear-encoded single gene copy loci, the microsatellite locus, and the two organellar loci (Supplemental Figure 1). To differentiate between alleles undergoing minor mutational drift, from those that have evolved independently as distinct genetic outgroups, 1000 bootstrap replicates were run for each tree, and supported nodes above $60 \%$ are indicated at each marker. For each tree, the 19 isolates identified as Type $\mathrm{X}$ were labeled in purple and isolates belonging to the clonal lineage I $(n=1)$, II $(n=3)$, and III $(n=1)$ were labeled in red, green, and blue respectively (Figure 2).

At 5 genetic markers located on chromosomes Ib, II, III, XI, and XII, all 12 distinct Type $\mathrm{X}$ haplotypes possessed a canonical Type II allele (green), with no minor mutational drift detected, consistent with Type II being one of the parental genetic backgrounds (Supplemental Table 1). At the L358 marker on chromosome V, however, all Type X isolates 
except 3675 possessed a Type I or " $\alpha$ " allele (red), indicating the presence of mixed ancestry. Further, at the BSR4 marker on chromosome IV, all Type X isolates except 3675 possessed an entirely novel allele that was readily differentiated from Type I, II and III strains with strong bootstrap support (Figure 2B). At all remaining loci, Type X isolates possessed either a Type II or one of 2 genetically distinct alleles, which could not be readily explained by minor mutational drift. Hence, across the 17 nuclear-encoded markers, each isolate possessed either a Type II allele, or an allele of distinct ancestry that was referred to as belonging to the " $\alpha$ " (red), " $\gamma$ " (purple) or " $\delta$ " (orange) lineages.

The maternally inherited organellar genome markers were likewise mixed. At the mitochondrial marker C01, two alleles were identified, either an allele common to Type I, II and III strains or a novel allele designated " $\gamma$ " (Figure 2A). Further, 3 distinct alleles were identified at the apicolast marker APICO: a Type I or " $\alpha$ " allele (red), a Type II allele (green) or a novel " $\gamma$ " allele (purple), supporting the mixed ancestry designation. Each of the 12 Type $\mathrm{X}$ haplotypes were therefore a mosaic of mixed ancestry that possessed some combination of Type II, Type I, " $\gamma$ " or " $\delta$ " alleles that had segregated independently across the loci investigated. The data are consistent with Type X existing as a recombinant clade resembling F1 progeny that was recently derived, without sufficient time to develop minor mutational drift (Supplemental Table 1).

In support of the sexual cross model, incongruity between phylogenies for each haplotype was readily observed between markers located in different portions of the genome. In Figure 2C, 8 isolates shared the same ancestry between the two genetic loci located on separate chromosomes VIIa and VIIb (GRA7 and BAG1 respectively) and were shaded either green (Type II alleles at both loci) or purple ( $\gamma$ lineage alleles at both loci). In contrast, 13 isolates had independently segregated chromosomes of mixed ancestry, colored orange and pink (to depict isolates that were discordant and possessed different ancestral alleles at each locus), and the crossing of orange and pink lines highlighted this lack of congruence (Figure 2C). For example, isolate 3671, had a $\gamma$ lineage allele at GRA7 but a $\delta$ lineage allele at BAG1. Furthermore, recombination within a chromosome was likewise observed at linked markers for 18 strains (Figure 2D). For example, isolate 3387, had a $\gamma$ lineage allele at GRA7 but a $\delta$ lineage allele at SAG4, whereas 3675 had a II lineage allele GRA7 but a $\gamma$ lineage allele SAG4, formally establishing that recombination had occurred and supporting genetic hybridization as the most plausible explanation for the origin of the 12 Type X haplotypes.

\section{Type $\mathrm{X}$ is an admixture cross between Type II and a novel genetic background by CGH- array hybridization}

A subset of Type X isolates, 2 each from haplotypes A (3142, 3265), C (3026, 3045), F $(3503,3429)$ and one each from $D(3178)$ and $H$ (3671) were next hybridized against a photolithographic microarray that possessed 1517 polymorphic Type I, II and III strainspecific genotyping probes distributed genome-wide [37]. This was done to test whether the MLST genetic markers accurately predicted the presence of large genome-wide haplotype specific blocks, consistent with genetic hybridization. Each Type-specific SNP on the CGH array is represented as a dot colored either red, green or blue respectively for a hybridizing strain that possesses a Type I, II or III SNP at the probe position. Dots colored grey only identify probes that failed to hybridize, consistent with a strain that does not possess a Type 
323 I, II or III specific SNP at the probe position. Hybridization with DNA from a Type I (GT1), Type II (Me49), and Type III (CTG) strain identified hybridization patterns consistent with genotype, indicating that all genotype-specific probes were functioning as expected (Figure 3A). Hybridization with the Type $\mathrm{X}$ isolates identified large, contiguous Type II haploblocks, as evidenced by hybridization of all Type II-specific probes present on Chr III, VI, VIIb and XII, for example. However, in other portions of the genome, the SNP diversity pattern was novel. The hybridization pattern in these regions either shared patch-work similarity with Type I (i.e., at Chr Ib, VI) or was highly divergent with large contiguous blocks of SNP probes colored grey that failed to hybridize, which was consistent with introgression of a non-Type I, II or III genetic ancestry, rather than minor mutational drift as the explanation for lack of reactivity (i.e., Chr IV, XI, right end of Chr VII, XII, left end of Chr VIII).

The pattern of hybridizing SNPs was unique for each of the seven Type X isolates examined, but it was only minimally different; either 3, 5, or 15 SNP differences were detected in pairwise comparisons within haplotype A $(3142,3265), C(3026,3045)$, and F (3503, 3429), respectively (Figure 3A). At this resolution, it was not possible to distinguish between CGH array hybridization efficiency, from that of minor mutational drift. Indeed, the CGH arrays each had approximately the same number of SNP differences (3-15) between haplotypes as they did within a haplotype, which is more consistent with hybridization efficiency as the probable explanation for these hybridization differences. It did, however, establish that 3671 is a distinct genetic admixture because it possessed a different Type II hybridization pattern at Chr Ia, left hand of Chr Ib, VI, XI and right side of Chr XII, which was distinct from all other Type X strains analyzed (Figure 3A).

\section{Type $\mathrm{X}$ is a recombinant clade at WGS resolution}

The lack of resolution using the CGH array approach indicated that WGS was required to infer an accurate genetic history model for the Type $\mathrm{X}$ strains. Using genome-wide polymorphism data derived by identifying all variant SNP positions after reference mapping a Type I, II, III, 16 Type X, and three previously WGS sequenced HG12 isolates (WTD-1, RAY, ARI) against the published Me49 genome, we generated an unrooted Neighbor-Net tree (Figure 3B). The Neighbor-Net analysis established that all Type X isolates, in similar to Type I and III strains, shared blocks of genome-wide ancestry with Type II, represented by the reticulated pattern of edge blocks that depict recombination events between Type II and the different genetic backgrounds within X, I and III. It did not support a clonal lineage designation as the majority of Type $\mathrm{X}$ isolates appeared on separate branches (Figure 3B, Inset). Only clonal complexes 1 and 4 (CC1, Type II; CC4, 3045, 3160, 4167) were supported at WGS resolution, and 3 previously sequenced HG12 isolates from 2 people (ARI, RAY) and a deer (WTD-1) formed well-supported clades with Type X isolates 4166 (WTD-1; RAY) and 3168 (ARI) indicating that Type $\mathrm{X}$ has a wider distribution beyond sea otter infection. Outside of the reticulated network of edge blocks, the branch length for each Type X isolate was significantly less than that observed for Type I and III, synonymous with a more recent origin, without sufficient time to accumulate private SNPs by mutational drift. When murine virulence data for each genotype was mapped onto the Neighbor-Net tree (see inset, Figure 3B), a clear partitioning of the different virulence phenotypes was resolved along branches within the network, consistent with Type $\mathrm{X}$ isolates existing as sister progeny with only minor mutational drift detected at each supported branch. 
To identify the size, genome distribution, and total number of Type II admixed haploblocks introgressed into each Type X isolate, pairwise SNP diversity plots were generated for all Type X isolates, as well as reference Type I and III strains that have been previously shown to have recombined with Type II. The Type I GT1 strain possessed an Me49 Chr Ia and IV, and an Me49 admixture block at the left side of Chr VIIa, and right end of Chr XI (Figure 4). As expected, the Type III VEG strain possessed many more Me49 admixture blocks distributed genome-wide than GT1, comprising 40\% of its genome. Only Type X haplotype $H$ (3671) possessed haploblocks that were highly similar in sequence to Me49, on Chr Ia, II, III, VI, VIII, IX, XI, and XII (Figure 4). In other regions, however, 3671 shared regions clearly Me49-like, but that appeared to have diverged somewhat by mutational drift (average 3-5 SNPs per 10kb block) on Chr II or introgressed into Chr IV, V, VIIa, VIIb, VIII, IX, and XII. Intermixed within the Type II regions of the 3671 genome were a limited number of large haploblocks containing divergent SNP density synonymous with hybridization by a strain possessing distinct $\gamma$ or $\delta$ genetic ancestry (average of 50-100 SNPs per 10kb block). When the pairwise SNP density analysis was expanded to different Type X haplotypes, isolates within each haplotype possessed highly similar patchwork mosaics of Type II-like or divergent (Type X) haploblocks that were specific to each haplotype. For example, haplotype A isolates 3142 and 3168 SNP density plots were highly similar to each other, and to haplotype C isolate 3045, but were readily distinguishable from isolates 4166 and 3503, strains within haplotypes D and F, respectively (Figure 4). Specifically, isolate 3142 had a Type X haploblock inheritance pattern at the right end of Chr V, and in the middle of Chr Ib, VIIa, VIII, IX and XII whereas 4166, which was indistinguishable from a previously sequenced HG12 strain (RAY) recovered from a human patient, possessed either Type II or 3671 haploblocks in these regions (Figure 4). Although the haplotype F isolate 3503 was highly similar in genomic organization to 3142, it possessed a Type II haploblock at the right end of $\mathrm{Chr} \mathrm{V}$ that readily distinguished it from haplotype A. The pairwise SNP analysis established that the majority of Type $\mathrm{X}$ haplotypes possess a genomic architecture that is strikingly similar, but different at a limited number of admixture blocks. Coupled with the low allelic diversity, the data support a genetic history model whereby Type $\mathrm{X}$ resembles a sexual clade of recently-derived natural recombinants from a relatively limited number of crosses.

\section{PopNet analysis identifies only limited admixture blocks among Type $\mathrm{X}$ isolates}

While the Neighbor-Net analysis established that Type X exists as a recombinant clade of strains, it failed to predict the precise number of genetic ancestries, or how they had admixed positionally across the chromosomes. PopNet was used to paint chromosomes according to their local inheritance patterns [38]. Included in the analysis were all sequenced Type X and II strains, as well as reference Type I and III strains, known to have admixed with Type II. PopNet identified 4 statistically supported ancestries and showed that each genome was a mosaic of these distinct ancestries, supporting the admixture model.

Within the circle depicting the Type $\mathrm{X}$ clade, 5 distinct subgroupings were identified based on the number and position of shared ancestral blocks; these were grouped together based on line thickness (Figure 5A). These same groupings were supported both by Pairwise SNP plots and Neighbor-Net analysis, but the PopNet analysis showed which of the 5 ancestries had introgressed positionally across the mosaic genomes. Clear recombination blocks were readily resolved, and the genome architecture was remarkably similar between the groupings. A custom script designed to identify only major crossover points between the 
two parents (Type II and the mosaic ancestry of the $\gamma / \delta$ parent) identified either a limited number of single and double recombination events, or the inheritance of whole chromosomes of either Type II (Chr II) or $\gamma / \delta$ (Chr Ia, IV, XI) parental ancestry, consistent with this group of strains representing related sister progeny (Figure 5B). Because murine virulence plotted on the Neighbor-Net tree identified clear pathogenicity differences in mice that clustered based on parasite genotype, and that the Type X strains resembled a natural clade of recombinant progeny (akin to $F_{1}$ ) that possessed only a restricted number of crossover points, a population-based QTL was performed to see if genes could be mapped that contribute to murine virulence within the set of 18 natural isolates for which virulence data existed (Figure 5C),

\section{Natural Population-based QTL identifies ROP33 (WNG-3) as a new murine Vir locus}

All Type $X$ isolates recovered from sea otters resembled offspring from one (or a few crosses) between a Type II strain, which is avirulent in mice, and unknown parent(s) that are mosaic for two distinct ancestries. Because these natural isolates possessed a range of virulence phenotypes when infected into mice (Figure 1C), a genome scan was performed to determine the log-likelihood for association of discrete genome haploblocks with the acute virulence phenotype. Four quantitative trait loci (QTL) peaks were identified with logarithm of odds (LOD) scores 4.0 or greater on chromosomes V, VIIa, VIII, and X (Figure 6A). The average size of the genomic regions spanned by the QTLs were in the range of $100-200 \mathrm{~kb}$, except for the one on chromosome $\mathrm{V}$, which was $>700 \mathrm{~kb}$ (Figure 6B). To identify candidate genes within the four peaks, the following inclusion criteria were assessed: presence of a signal peptide and/or transmembrane domain, gene expression and polymorphism differences, as well as every genes CRISPR genome-wide mutagenesis score for essentiality [39](Supplemental Table 2). ROP33 on chromosome VIIa stood out as the best candidate gene to target for reverse genetics as it was abundantly expressed during acute infection, it was predicted to be a functional serine-threonine protein kinase, it was highly polymorphic, and is part of a family of divergent WNG (with-no-Gly-loop) kinases that regulate tubular membrane biogenesis in the parasite [33]. Further, one allele of ROP33 was strongly correlated with acute virulence $(\mathrm{p}=0.00031$; fishers two-sided exact test) (Figure 6C).

To determine whether ROP33 was a novel murine virulence gene, the ROP33 locus was disrupted by targeted deletion using CRISPR-Cas 9 facilitated double crossover homologous

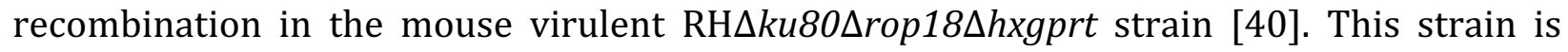
virulent in mice ( $\mathrm{LD}_{100}=500$ tachyzoites) and was engineered to accept targeted replacement of the rop33 gene using an HXGPRT gene flanked by $30 \mathrm{bp}$ of homology just outside of the rop33 promoter and 3'UTR region. Following selection in mycophenolic acid (MPA) and xanthine (to select for the HXGPRT gene), the population was screened for disruption of the

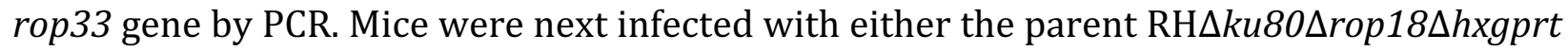
strain or the Rop33- mutant to assess murine virulence. Groups of 5 outbred CD-1 mice were injected intraperitoneally with 500 tachyzoites and the results shown are for one of two independent experiments. All mice infected with the parent $\mathrm{RH}$ line succumbed to infection within 20 days (Figure 6D). In contrast, targeted deletion of the rop33 gene was protective, the majority of mice survived acute infection, and our results suggest that ROP33 is a novel 
detected through acute infection by bioluminescence imaging, indicating that deletion of ROP33 does not appear to affect parasite proliferation in vivo (Figure 6E). All surviving mice were confirmed to be positive by serology, indicating that they had been productively infected. These data strongly suggest that ROP33 is a new virulence factor for murine infection that was identified using natural isolates derived from a population genetic cross performed in nature.

\section{Discussion}

Here, we investigated the emergence of a presumed clonal lineage of Toxoplasma gondii (Type X; also referred to as HG12) recovered from a single marine host, infected southern sea otters in California, that were collected longitudinally over a 7 year period in order to determine the genetic basis for this epizootic in a federally listed, threatened species. Although Type $X$ has previously been identified as the $4^{\text {th }}$ clonal lineage in North American wildlife, work done here established that Type X is not a clonal lineage, but rather a recombinant clade of strains that resemble $F_{1}$ progeny from at least one natural cross. DNA sequence analysis using a wide selection of linked and unlinked markers across the nuclear and organellar genomes of Type $\mathrm{X}$ isolates identified Type $\mathrm{X}$ to be a composite of Type II and an unknown mosaic of two distinct ancestries, referred to as $\gamma$ and $\delta$, that have recombined to produce a highly invasive clade of strains causing both subclinical infection as well as mortality in threatened marine mammals, including the southern sea otter. Our data suggest that Type X was derived from one or a limited number of crosses from two highly related parental strains. One sea otter genotype was widely distributed and found to have expanded to cause the majority of subclinical infections, but it was highly virulent to mice. Our natural population dataset supports a model whereby Toxoplasma's sexual life cycle is facilitating the evolution and expansion of cryptically virulent strains that are capable of causing pathogenic disease in a host-species specific manner. Hence, for generalist parasites that have broad host ranges, natural selection among intermediate hosts appears to maximize transmission and establishes how virulent strains can be maintained cryptically in nature. The expansion of a mouse virulent clone into a new ecological niche, such as near-shore marine mammals of the Eastern Pacific coastline, demonstrates how Toxoplasma gondii is leveraging its intermediate host range to selectively partition parasite genetic diversity and epidemic disease potential.

In previous studies, unknown strains having a distinct ancestry, referred to as $\alpha$ and $\beta$, were found to have crossed with Type II to create the Type I and Type III clonal strains respectively [11]. The frequency and pattern of recombined blocks inherited within the Type $\mathrm{X}$ strains is parsimonious with previously described models for the genetic history of Types I and III in which an unidentified ancestor (respectively $\alpha$ and $\beta$ ) sexually recombined with an ancestral Type II to create the new lineages (Figure 5B) [11,41]. Additionally, it has been suggested previously that Type $\mathrm{X}$ is a product of genetic hybridization between a Type II lineage and an unknown $\gamma$ lineage $[25,27,28,32,42]$. Here, we have further refined this perspective and concluded that Type $\mathrm{X}$ is a mosaic of a cross between Type II strains and two distinct $\gamma$ and $\delta$ ancestries. The $\gamma / \delta$ lineage has likely recombined at least once with Type II to create the Type X recombinant clade of strains. Currently, no single isolate has been found with Type II to produce the clonal lines I and III, respectively [11, 41]. 
Previous work on Type $\mathrm{X}$ circulating in wildlife samples using a limited set of genotyping markers identified Type $X$ as the $4^{\text {th }}$ clonal lineage in North America $[10,27]$. However, this designation was based on the discovery of genetically distinct alleles at only two loci, GRA6 and GRA7, which was the original basis for distinguishing the Type X lineage as a new genotype from that of Type II, and that it had undergone a hybridization event with a novel genotype [25]. It is now well established that Type X commonly infects wildlife across North America $[10,14,29,30,32,36]$. This study examined Toxoplasma strains collected longitudinally across a 7 year period and large geographic range from sea otter infections, and it was comprised of infections ranging from asymptomatic to subclinical or considered the primary cause of death. As previous work had classified Type X as clonal, finding such a myriad of different disease states among infected sea otters was not expected $[10,27,32$, 36]. To ascertain whether parasite genotype was a critical parameter influencing the spectrum of disease, genotyping studies were carried out using an expanded set of markers to ascertain the true population genetic structure of the isolates belonging to Type X. All genotyping markers used in this study were sequenced to increase resolution rather than evaluated strictly for their PCR-RFLP genotype, as was the standard for many of the previous studies. While the markers used herein only surveyed a small part of the genome, they identified at least 12 distinct haplotypes within Type X, confirming that they do not exist as a clonal population, as hypothesized in another study [26]. Furthermore, at any given locus, only a Type II allele or one or another of two distinct alleles was identified indicating limited allelic diversity. Hence, each of the 12 haplotypes possessed one of three allelic types at any one locus that had independently segregated across the markers examined. This result is parsimonious with a recombinant clade of strains that reflect genetic hybrids from a limited, but distinct set of ancestries. Our results were further confirmed both by CGH array analyses as well as whole genome sequencing. The previous misclassification as a clonal population appears to be the result of the low resolution genotyping analyses performed, and the placement of the markers in predominantly Type II regions of the genome [10, 14, 43]. For this reason, future studies should use WGS to not only discriminate between haplogroups within a population but also to determine the true genetic ancestry of each haplogroup. Toxoplasma population genetics would also greatly benefit from an increase in the number of isolates that are whole genome sequenced. WGS should be expanded to include both a greater diversity of strains as well as multiple isolates from within a canonical clonal lineage.

The Type I and III clonal lineages are thought to be derived from a limited number of sexual crosses, and all strains within these clonotypes share large haploblocks of Type II-like sequence [11,42, 44, 45]. This finding also holds true for the Type X lineage. At all loci surveyed, Type $\mathrm{X}$ displayed at least one isolate with a Type II inherited allele, and the introgression of large haploblocks of Type II ancestry across the genomes was confirmed in the 19 strains that were resolved at WGS resolution. In regions that did not clade with Type II, two different allelic types were identified. We concluded that these represented distinct ancestries that we referred to as $\gamma$ and $\delta$. This designation was supported by the inherent reticulation of the clade, seen in the NeighborNet tree (Figure 2B), which possessed short branch lengths radiating from a reticulated network with multiple strains on a single branch, rather than producing a star-like phylogeny with multiple alleles present at each major branch, the result of private SNPs that accumulated through genetic drift. In fact, the lack of genetic drift between these loci supported genetic hybridization as the most parsimonious explanation for the relationship between the Type $\mathrm{X}$ haplotypes. 
Sexual replication with genetic recombination is known to occur in South America at high frequency $[41,44,46]$. Additionally, recent studies on the population genetic structure of Toxoplasma has established that genetic hybridization, at whole genome resolution, is extant for the vast majority of sequenced strains that represent the breadth of genetic diversity within Toxoplasma $[15,38]$. Evidence of sexual recombination across the population, as well as the evidence of sexual replication within the Type X clade shown here, may indicate that sexual recombination is occurring more frequently than previously suspected within North America.

No barriers appear to exist, or have been described, to limit sexual recombination from occurring in the laboratory setting, although there is a lack of understanding for the genetic factors that regulate sexual replication in the felid host [7, 45, 47-49]. It is not clear why sexual recombination does not appear to be occurring in North American wildlife, to the degree that it does in South America, although it is possible that self-mating within the population masks the detection of genetic outcrossing $[12,42,50]$. Especially among closely related isolates, unisexual mating cannot be resolved using current genotyping methods that rely on low-resolution analyses and thus cannot readily distinguish asexual expansion from unisexual mating [12].

Toxoplasma has been shown previously to utilize its sexual cycle to expand its biological potential and alter its pathogenesis [7, 47, 49]. Sexual recombination, by the ability to reshuffle parental alleles into new combinations, can produce progeny with a vast range of altered biological potentials [7]. Our work herein has identified a single Toxoplasma genotype that was derived from a recent genetic cross that has been naturally selected in southern sea otters across a space of 7 years. Importantly, this same genotype was highly pathogenic to outbred laboratory mice, with all mice dying in 10-15 days with an inoculum of just 50 tachyzoites. In contrast, the two Type X B haplotype isolates that caused subclinical infection in outbred laboratory mice were highly pathogenic to sea otters that stranded with a fatal meningoencephalitis [25]. Our data thus support a virulence shift model for the expansion and propagation of Toxoplasma in nature, whereby Toxoplasma utilizes its sexual cycle to generate a wide spectrum of novel genotypes that possess altered biological potentials that can be naturally selected across Toxoplasma's vast host range for optimal transmission. Hence, natural selection among intermediate hosts allows Toxoplasma to maintain highly pathogenic strains cryptically that can cause serious disease in some animal hosts, but not in others. Examples of this type of selection have been observed previously in laboratory mice that express variable IRG (Immunity Related GTPase) gene arrays. IRGs are host proteins primarily responsible for combatting Toxoplasma lysis of murine cells and Toxoplasma encodes a suite of highly polymorphic Rhoptry kinase genes (ROPKs) whose primary function is to inactivate host IRGs [51-53]. While laboratory mice are highly clonal, wild mice and their subsequent IRGs are more diverse, as are the ROPKs expressed by different Toxoplasma strains that are capable of infecting wild mice $[17,54]$. Similarly, TLR $11 / 12$ is one of the primary rodent innate immune response sensors (otherwise referred to as PAMPs) that detects infection by Toxoplasma. However, not all intermediate hosts share a functional combination of TLR11/12, thus, Toxoplasma strains that are selected for infection in mice need to bypass TLR11/12 recognition, whereas this is not a barrier to infection in a human cell, for example [54,55]. Thus, intermediate hosts are capable of 594 naturally selecting Toxoplasma strain genotypes that establish chronic, transmissible 
a balance between its virulence, fitness and infectious transmissibility across its wide host range. It is this capacity to maintain cryptically virulent strains that may in part explain Toxoplasma's success in nature; it has the ability to cause disease outbreaks, expand its host range into new ecological niches, or alter its pathogenicity in both a parasite strain and host genetic manner to maximize its transmission.

In other protozoan parasites, when host partitioning occurs, it is synonymous with speciation. For example, Plasmodium parasites often partition by the mosquito host species they co-evolve with to maximize parasite transmission [56-60]. Likewise, the apicomplexan parasite Sarcocystis largely maintains separate species for each intermediate-definitive host species combination to maintain its life cycle $[12,61]$. In contrast, Toxoplasma forms transmissible cysts in virtually all warm-blooded vertebrates, and these cysts are infectious for its definitive felid host, as well as a large array of intermediate hosts. What is different, is that across the genetic diversity of Toxoplasma, specific strain genotypes are being selectively expanded among its vast array of different intermediate hosts, based primarily on parasite genotype and the suite of polymorphic effector proteins encoded by a particular strain. It is this fact that allows Toxoplasma to maintain cryptically virulent strains across its broad intermediate host range. Hence, the intermediate host is playing a central role in the natural selection, expansion, and maintenance of virulent strains across the broad host range of this generalist parasite. In effect, infected hosts act as reservoirs for the pathogenic or epidemic potential of the species. Our study showed that one sub-type of Toxoplasma had expanded in the majority of sea otters because it caused primarily subclinical, chronic infections in sea otters, but that it was uniformly lethal to outbred laboratory mice. This is not unlike the expansion of strains bearing Type I alleles in birds that harbor asymptomatic infections but prove highly pathogenic to laboratory mice [62]. Additionally, while Type II isolates are commonly found infecting domestic livestock in North America, Type X is more common in sylvatic hosts, leading others to propose that separate cycles exist within the Toxoplasma population that overlap solely within the feline definitive host $[10,29,63]$.

Finally, only a limited number of crossovers were detected among strains WGS sequenced within the recombinant clade of Type $X$ haplotypes. Because the pedigree of one of the parents was known, we concluded that the isolates resembled $F_{1}$ progeny from a natural cross, which prompted us to perform a population-based QTL analysis on an unmanipulated, natural population of genetic hybrids. Taking advantage of a genome-wide, high resolution SNP map we had generated for all sequenced haplotypes, our analysis identified multiple punctate QTL peaks containing a limited number of candidate loci associated with differences in mouse virulence for reverse genetic follow-up. The serinethreonine protein kinase ROP33 stood out as the best candidate to influence pathogenicity, it was identified previously to be an active protein kinase that is both abundantly expressed and highly polymorphic. Further, ROP33 is a divergent WNG kinase (WNG-3) that is related to ROP35 (WNG-1), a critical regulator of tubular membrane biogenesis, the formation of the parasite intravesicular network (IVN) and the phosphorylation of dense granule proteins associated with IVN biogenesis [33]. In fact dense granule proteins GRA2 and GRA6 are required for IVN biogenesis, and IVN deficient parasites grow normally in vitro, but are attenuated in mouse virulence assays $[64,65]$. Other related ROP proteins, including ROP 5, 16,17 , and 18 are known to hijack host immune signaling pathways to alter Toxoplasma virulence during rodent infection. However, all Type X isolates investigated in our study, whether mouse virulent or avirulent, only expressed avirulent allele combinations of the 
known ROP 5/16/18 virulence factors, which is why we chose to knock-out the gene in a Ku80 deficient strain of Toxoplasma that was both mouse virulent, and deficient in ROP18. This is because no change in mouse virulence was reported when ROP33 was deleted in a wild-type RH strain background [66]. In addition, previous genetic mapping studies failed to identify the QTL for this locus, even though it is polymorphic between Type I, II and III strains for which genetic crosses have been performed. It is likely that the ROP5 and ROP18 virulence factors were dominant, and ROP33 is analogous to ROP17, another virulence factor that was only identified after the virulence enhancing capacity of ROP18 was removed [40]. Our results show that parasites deficient in ROP33 in the highly permissive RH $\Delta$ ku80 $\Delta$ rop 18 parent, were avirulent, whereas an infectious dose of 500 tachyzoites of the RH $\Delta$ ku80 $\Delta$ rop 18 parent genotype was uniformly virulent. Specifically how ROP33 contributes to murine virulence, what dense granule proteins it phosphorylates, the integrity of the IVN and what host immune signaling pathways are modified are being actively investigated. Future work will dissect the host or parasite factors that ROP33 targets to reduce or influence parasite transmission and pathogenicity. Our study establishes that it is possible to use natural isolates derived from a population genetic cross in nature to identify genetic loci associated with a specific quantitative phenotype.

\section{Materials and Methods}

\section{Parasite culture}

Fifty-three previously published T. gondii strains isolated from brain and/or tissue samples from stranded Pacific coast southern sea otters were provided by Dr. Patricia Conrad $[25,26]$. Toxoplasma strains were isolated at the Marine Wildlife Veterinary Care and Research Centre in Santa Cruz, CA, or at the University of California, Davis School of Veterinary Medicine. Cause of death (COD) was determined for each sea otter based on primary pathology reports from marine veterinarians at date of necropsy, as previously published [25, 26, 31, 67]. Toxoplasma isolates each had an ATOS ("as the otter swims") number assigned to them based on the stranding location where each sea otter was discovered. Isolates were named sequentially based on the date the sea otter host stranded. Parasites were cultured and maintained in human foreskin fibroblast (HFF) monolayers as described previously [68].

\section{DNA extraction and genetic typing markers}

Parasites were syringe lysed using a 27-gauge needle and filtered through a 3.0 micron polycarbonate filter to remove cellular debris. DNA was extracted from cell pellets using the Qiagen DNeasy Blood and Tissue kit (Qiagen). All 53 isolates were typed using 5 previously described PCR-RFLP and one DNA sequencing marker [7, 25, 69-71]. BSR4, BAG1, and ROP1 were PCR amplified and typed based on known RFLP identity. GRA6 and SAG3 were typed according to previously identified representative SNPs based on Sanger sequence data done by the National Institute of Allergy and Infectious Disease's Rocky Mountain Laboratory (NIAID RML).

Alleles observed via sequencing were designated as canonical I, II, or III. When sequences differed from canonical alleles, previously described methods of characterization were used [29]. Briefly, when genetic variation differed from references by two mutations the allele was categorized as unique, while only one mutation difference was labeled a drifted 
688

689

690

691

692

693

694

695

696

697

698

699

700

701

702

703

704

705

706

707

708

709

710

711

712

713

714

715

716

717

718

719

720

721

722

723

724

725

726

727

728

729

730

731

732

allele from the closest reference sequence. When more than two nucleotide mutations were present, or the sequence was as distant from the reference strains as references are from each other, alleles were classified as novel alleles.

21 strains representing the 8 unique genotypes identified by the original 5 markers were selected to characterize further. These 21 strains were PCR amplified, sequenced, and genotyped using 20 markers: 18 at both linked and unlinked genomic loci, encompassing 13 of the 14 chromosomes, and 2 loci on organellar genomes (apicoplast and mitochondria). 15,430 bp with 335 SNPs $(\sim 0.024 \%$ of the genome). Sequences were examined and nucleotides verified using SeqMan Pro alignment software (Lasergene). Sequences for reference strains (ME49, GT1, and VEG) were downloaded from ToxoDB [72].

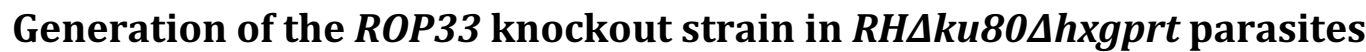

Deletion of the ROP33 gene was generated using the Type I parasite RH that was rendered deficient in the expression of KU80 and the drug selectable marker HXGPRT. Briefly, the CRISPR/Cas9 targeting plasmid pSAG1:CAS9,U6:sgUPRT (received from Prof. L. David Sibley, Department of Molecular Microbiology, Washington University School of Medicine) was modified with a guide RNA (GTCGGACGCGAAACTCGCTT) to target the 5' region of the ROP33 gene (sgROP33) using Q5 mutagenesis (New England Biolabs, MA). Then a CRISPR/Cas9 replacement construct was created using Gibson assembly (New England Biolabs) to stitch together a $1 \mathrm{~kb}$ flanking region of the 5' UTR region of ROP33 (TGGT1_chrVIIa, 3921773 to $3922773 \mathrm{bp}$ ) with the selectable marker HXGPRT and a 1kb flanking region of the 3'UTR region of ROP33 (TGGT1_chrVIIa, 3926189 to $3927189 \mathrm{bp}$ ) to replace the ROP33 gene using the selectable drug cassette. To delete the ROP33 gene, a total of $50 \mu \mathrm{g}$ of the sgROP33 and replacement construct (5:1 ratio) were co-transfected into a ROP18 deficient strain of RH (RH $\Delta k u 80 \Delta h x g p r t \Delta r o p 18$ ), which was cultured in human foreskin fibroblasts (HFF) cells and maintained in Dulbecco's modified Eagle's medium (DMEM) supplemented with 10\% fetal bovine serum, $2 \mathrm{mM}$ glutamine and $25 \mu \mathrm{g} / \mathrm{ml}$ gentamicin. After transfection, parasites were selected for stable integration of the targeting construct using mycophenolic acid (12.5 mg / $\mathrm{mL}$ in MeOH; MPA) / xanthine (20 mg / mL in $1 \mathrm{M} \mathrm{KOH} ; \mathrm{XAN}$ ) as described previously [73]. After 15 days, the resistant population was cloned by limiting dilution and single clones were screened by PCR for targeted deletion of the ROP33 gene.

\section{Mouse virulence assay}

To determine the virulence of the sea otter isolates in a mouse model, groups of five or more, 6-8 week-old, female CD1 outbred mice were intraperitoneally injected with $50 \mathrm{~T}$. gondii tachyzoites resuspended in $500 \mu \mathrm{l}$ of PBS that had been expanded in HFF cells. Mice were weighed daily to measure infection-induced cachexia and mouse survival was assayed over 42 days [7]. At 14 days, mice were bled, and serum extracted to test for seroconversion via indirect fluorescent antibody test (IFAT) against ME49 tachyzoites [74]. Strain virulence was classified as follows: avirulent strains killed no seropositive mice within 42 days of infection, intermediate virulent strains killed some but not all seropositive mice within 42 days post-infection, and virulent strains killed all seropositive mice. To assess the contribution of ROP33 to mouse virulence, groups of five 6-8 week old female outbred CD-1 mice were injected intraperitoneally with 500 tachyzoites of either the parent 


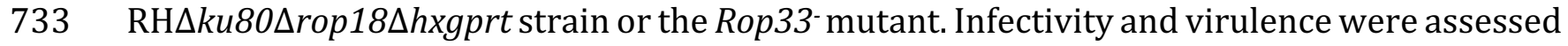

734 as above.

735

736

737

738

739

740

741

742

743

744

745

746

747

748

749

750

751

752

753

754

755

756

757

758

759

760

761

762

763

764

765

766

767

768

769

770

771

772

773

774

775

776

777

\section{Phylogenetic Tree and DNA Marker Analysis}

The default settings of Clustal X were used to align DNA sequences for all markers individually and as a concatenated set of markers [75]. MSF files of aligned marker sequences were imported into Molecular Evolutionary Genetic Analysis (MEGA) Version 7 to create a maximum likelihood tree using Tamura-Nei model distance analysis with uniform rates of substitution across all sites, and 1000 bootstrap support for all branch points [76]. Consensus trees were rooted on ME49, as Type II is an inherent parent in all strains analyzed. All scales are by 0.001 nucleotide difference unit. Distinct parental lineages with bootstrap support over $60 \%$ are indicated on each tree to distinguish II alleles from novel $\gamma$ and $\delta$ alleles. These novel alleles are indicated in purple and orange respectively on the 17 loci MLST in Supplementary Table 2. The Nexus file of the concatenated marker alignment was imported into SplitsTree 4 Version 4.13.1 where NetworkNet analysis was run using default settings [77].

\section{eBURST Clonal Complex Analysis}

Alleles determined from the 17 nuclear encoded markers (excluding ROP1 due to its classification as a microsatellite marker) were given numerical designations to create a multilocus sequence-typing scheme for the eBURST program [78]. Default settings were used to evaluate the isolated strains' alleles. Strains were displayed as dots and colors of dots were depicted accordingly: Type I, II, III, and X being red, green, blue, and purple respectively. Dot size was representative of the number of strains contained within each genotype, larger dots representing more strains bearing the same genotype. Strains sharing 16 of the 17 nuclear encoded markers were designated as clonal complexes and represented by connecting lines in the diagram shown.

\section{Genomic hybridization to Affymetrix arrays}

Genomic DNA was sheared and biotin labeled before hybridizing to a custom T. gondii Affymetrix microarray as previously published [37]. High-fidelity SNPs were characterized via a custom R script to identify SNPs belonging to each of the three reference strains (I, II, and III). Three reference strains (GT1, ME49, and CTG) are shown to demonstrate ideal hybridization within canonical lineages. Sea otter Type X DNA isolate hybridizations are shown below the reference strains.

\section{Whole-Genome Sequence Analysis of Type X Recombination}

DNA from 16 isolates that grew best in tissue culture as well as 3 representative Type $\mathrm{X}$ strains (ARI, RAY, and WTD1), for a total of 19 Type X strains was isolated. $3 \mu \mathrm{g}$ of DNA isolated from each of these 19 strains was sent to NIAID RML for whole-genome sequencing using Illumina HiSeq technology. Fastq reads were reference mapped to the T. gondii ME49 assemblage Version 8.2 [72] using BWA 0.7.5a to align the reads to the reference genome and GATK 3.7 in coordination with Picard 1.131 following best practices to quality control the mapped reads [80]. Following mapping, the gVCF method of GATK was used to combine SNP calls using stand_call_conf of 30.0, nct of 10, and ploidy of 1 (for haploid genomes) to 
call 568,592 single nucleotide polymorphism positions across the whole genomes of these strains [80]. The derived VCF formatted SNP file was curated using GATK and VCFTools to produce a tabular file containing only biallelic SNPs with no large insertions or deletions [81]. A custom script was then utilized to convert this SNP file into a fasta file of strain polymorphic positions across the Type $\mathrm{X}$ and reference genomes. These SNP fasta files were run in SplitsTree4 using default parameters for BioNJ with 1000 bootstrap support to create a NeighborNet tree [72]. Interconnected reticulation between strains is indicative of recombination between strains while mitotic drift was visualized by divergent branching.

\section{SNP Density Fingerprint Analysis of Recombinant Progeny}

The same tabular, biallelic SNP file used in the NeighborNet analysis was modified using a custom $\mathrm{R}$ script based on location mapping to isolate strain-specific polymorphic locations from the tabular VCF. Further R scripts group these SNPs into $100 \mathrm{~kb}$ windows which are mapped across the genomes of these strains to display where diversity from the reference genome (ME49) is most apparent [44, 82]. The larger the number of SNPs in a particular window, the more divergent the strain is from the ME49 reference. Distinct haploblocks where genomic recombination has occurred are apparent in areas where haploblock diversity significantly varies from the surrounding regions on the same chromosome.

\section{PopNet Characterization of Strain Interrelatedness}

The tabular, biallelic SNP file created from WGS of Type X and used to derive the SNP density plots and NeighborNet tree was uploaded into PopNet using default parameters to assess the diversity and interrelatedness of strains [38]. Cytoscape was used to visualize the recombination and Markov clustering outputs. Genomes are displayed in circularized format with chromosomes concatenated into a circular genome display. The background of each strain is painted to match the group that shares the most common ancestry over the entire genome with the strain shown. Chromosome painting was done in $10 \mathrm{~kb}$ increments and the sequence haploblock was painted based on its shared ancestry. For instance, a Type X isolate haploblock that was most closely related to the ancestral Type II strain was painted green to indicate its inheritance. Strains that are more closely related have thicker connecting lines between the circles of the strain isolates.

\section{Virulence Gene Identification by QTL}

To find novel virulence alleles, the tabular, biallelic SNP file was utilized to run a quantitative trait locus (QTL) analysis on the Type X and Type II isolates of Toxoplasma sequenced at WGS resolution [83]. SNP calls were down-selected by a custom java script to include one SNP every $5 \mathrm{~kb}$. This allows the QTL software to analyze the breadth of the WGS data in QTL, which was built to handle marker typing data without the depth inherent in WGS data. Custom scripts code the SNPs into reference (ME49) versus alternative (Type $X^{\prime}$ 's $\gamma / \delta$ lineage which substitute as the secondary parent) alleles. This down-selected marker dataset was combined with previously determined low-dose murine virulence for these strains. This data was then input into J/qtl and a one QTL genome scan was run using the default settings for the EM algorithm (maximum likelihood) with 1000 permutations to 
822 identify genomic locations significantly associated with the percentage of murine death due

823 to Type X infection [84].

824 The QTL calculations identified four significantly associated regions based on a LOD score of greater than 4.0. Within these genomic regions, 450 genes were predicted based on ToxoDB documentation. These potential genes were down-selected based on presence of a signal peptide and/or transmembrane domain, gene expression, polymorphism, and genome-wide CRISPR score for essentiality to identify 32 virulence candidate genes within the Type X strains. Of these, ROP33 was selected for further interrogation based on its high LOD score and similarity to previously identified virulence effector proteins.

\section{Funding statement:}

833

This work was supported in part by the Intramural Research Program of the National Institute of Allergy and Infectious Diseases (NIAID) at the National Institutes of Health and by the National Science Foundation, Ecology of Infectious Diseases Grant 052576.

\section{Ethics statements:}

The animal study protocol LPD 22E was reviewed and approved by the Animal Care and Use Committee of the Intramural Research Program of the National Institute of Allergy and Infectious Diseases, National Institutes of Health. No human studies or identifiable human data are presented in this study. All datasets generated for this study are available upon request to the corresponding author.

\section{Acknowledgements}

We thank Andrea Packham and Ann Melli for their assistance in culturing and isolation of the Toxoplasma isolates, and Javi Zhang for his help with PopNet analysis. We also thank all volunteers and staff at the California Department of Fish and Wildlife, the Monterey Bay Aquarium, the Marine Mammal Center, and the United States Geological Survey for their efforts to recover stranded sea otters that facilitated the isolation of Toxoplasma gondii strains utilized in this paper.

851

\section{References}

1. Vijaykrishna D, Smith GJ, Pybus OG, Zhu H, Bhatt S, Poon LL, et al. Long-term evolution and transmission dynamics of swine influenza A virus. Nature. 2011;473(7348):519-22. doi: 10.1038/nature10004. PubMed PMID: 21614079.

2. Bellanger X, Payot S, Leblond-Bourget N, Guedon G. Conjugative and mobilizable genomic islands in bacteria: evolution and diversity. FEMS Microbiol Rev. 2014;38(4):720-60. doi: 10.1111/1574-6976.12058. PubMed PMID: 24372381.

3. Schneider G, Dobrindt U, Middendorf B, Hochhut B, Szijarto V, Emody L, et al. Mobilisation and remobilisation of a large archetypal pathogenicity island of uropathogenic Escherichia coli in vitro support the role of conjugation for horizontal transfer of genomic islands. BMC Microbiol. 2011;11:210. doi: 10.1186/1471-2180-11210. PubMed PMID: 21943043; PubMed Central PMCID: PMC3202238.

4. Wardal E, Markowska K, Zabicka D, Wroblewska M, Giemza M, Mik E, et al. Molecular analysis of vanA outbreak of Enterococcus faecium in two Warsaw hospitals: the importance of mobile genetic elements. Biomed Res Int. 2014;2014:575367. doi: 
10.1155/2014/575367. PubMed PMID: 25003118; PubMed Central PMCID: PMC4070583.

5. English ED, Adomako-Ankomah Y, Boyle JP. Secreted effectors in Toxoplasma gondii and related species: determinants of host range and pathogenesis? Parasite immunology. 2015;37(3):127-40. doi: 10.1111/pim.12166. PubMed PMID: 25655311.

6. Fraser JA, Giles SS, Wenink EC, Geunes-Boyer SG, Wright JR, Diezmann S, et al. Same-sex mating and the origin of the Vancouver Island Cryptococcus gattii outbreak. Nature. 2005;437(7063):1360-4. Epub 2005/10/14. doi: 10.1038/nature04220. PubMed PMID: 16222245.

7. Grigg ME, Bonnefoy S, Hehl AB, Suzuki Y, Boothroyd JC. Success and virulence in Toxoplasma as the result of sexual recombination between two distinct ancestries. Science. 2001;294(5540):161-5. doi: DOI 10.1126/science.1061888. PubMed PMID: WOS:000171448800052.

8. Boothroyd JC, Grigg ME. Population biology of Toxoplasma gondii and its relevance to human infection: do different strains cause different disease? Curr Opin Microbiol. 5. England2002. p. 438-42.

9. Boyer K, Hill D, Mui E, Wroblewski K, Karrison T, Dubey JP, et al. Unrecognized ingestion of Toxoplasma gondii oocysts leads to congenital toxoplasmosis and causes epidemics in North America. Clin Infect Dis. 2011;53(11):1081-9. Epub 2011/10/25. doi: 10.1093/cid/cir667. PubMed PMID: 22021924; PubMed Central PMCID: PMCPMC3246875.

10. Behnke MS, Khan A, Wootton JC, Dubey JP, Tang K, Sibley LD. Virulence differences in Toxoplasma mediated by amplification of a family of polymorphic pseudokinases. Proc Natl Acad Sci U S A. 2011;108(23):9631-6. doi: 10.1073/pnas.1015338108. PubMed PMID: 21586633; PubMed Central PMCID: PMC3111276.

11. Boyle JP, Rajasekar B, Saeij JP, Ajioka JW, Berriman M, Paulsen I, et al. Just one cross appears capable of dramatically altering the population biology of a eukaryotic pathogen like Toxoplasma gondii. Proc Natl Acad Sci U S A. 2006;103(27):10514-9. doi: 10.1073/pnas.0510319103. PubMed PMID: 16801557; PubMed Central PMCID: PMC1502489.

12. Wendte JM, Miller MA, Lambourn DM, Magargal SL, Jessup DA, Grigg ME. Self-mating in the definitive host potentiates clonal outbreaks of the apicomplexan parasites Sarcocystis neurona and Toxoplasma gondii. PLoS Genetics. 2010;6(12):e1001261. doi: 10.1371/journal.pgen.1001261. PubMed PMID: 21203443; PubMed Central PMCID: PMC3009688.

13. Boothroyd JC. Expansion of host range as a driving force in the evolution of Toxoplasma. Mem I Oswaldo Cruz. 2009;104(2):179-84. PubMed PMID: WOS:000267051500009.

14. Howe DK, Sibley LD. Toxoplasma gondii comprises three clonal lineages: correlation of parasite genotype with human disease. J Infect Dis. 1995;172(6):1561-6. PubMed PMID: 7594717.

15. Lorenzi H, Khan A, Behnke MS, Namasivayam S, Swapna LS, Hadjithomas M, et al. Local admixture of amplified and diversified secreted pathogenesis determinants shapes mosaic Toxoplasma gondii genomes. Nat Commun. 2016;7:10147. doi: 10.1038/ncomms10147. PubMed PMID: 26738725; PubMed Central PMCID: PMCPMC4729833. 
16. Sibley LD, Boothroyd JC. Virulent strains of Toxoplasma gondii comprise a single clonal lineage. Nature. 1992;359(6390):82-5. doi: 10.1038/359082a0. PubMed PMID: 1355855.

17. Lilue J, Muller UB, Steinfeldt T, Howard JC. Reciprocal virulence and resistance polymorphism in the relationship between Toxoplasma gondii and the house mouse. Elife. 2013;2:e01298. doi: 10.7554/eLife.01298. PubMed PMID: 24175088; PubMed Central PMCID: PMC3810784.

18. Steinfeldt T, Konen-Waisman S, Tong L, Pawlowski N, Lamkemeyer T, Sibley LD, et al. Phosphorylation of mouse immunity-related GTPase (IRG) resistance proteins is an evasion strategy for virulent Toxoplasma gondii. PLoS Biology. 2010;8(12):e1000576. Epub 2011/01/05. doi: 10.1371/journal.pbio.1000576. PubMed PMID: 21203588; PubMed Central PMCID: PMCPMC3006384.

19. Cavailles P, Sergent V, Bisanz C, Papapietro O, Colacios C, Mas M, et al. The rat Toxo1 locus directs toxoplasmosis outcome and controls parasite proliferation and spreading by macrophage-dependent mechanisms. Proc Natl Acad Sci U S A. 2006;103(3):744-9. Epub 2006/01/13. doi: 10.1073/pnas.0506643103. PubMed PMID: 16407112; PubMed Central PMCID: PMCPMC1334643.

20. Cirelli KM, Gorfu G, Hassan MA, Printz M, Crown D, Leppla SH, et al. Inflammasome sensor NLRP1 controls rat macrophage susceptibility to Toxoplasma gondii. PLoS Pathog. 2014;10(3):e1003927. Epub 2014/03/15. doi: 10.1371/journal.ppat.1003927. PubMed PMID: 24626226; PubMed Central PMCID: PMCPMC3953412.

21. Ewald SE, Chavarria-Smith J, Boothroyd JC. NLRP1 is an inflammasome sensor for Toxoplasma gondii. Infection and Immunity. 2014;82(1):460-8. Epub 2013/11/13. doi: 10.1128/IAI.01170-13. PubMed PMID: 24218483; PubMed Central PMCID: PMCPMC3911858.

22. Gorfu G, Cirelli KM, Melo MB, Mayer-Barber K, Crown D, Koller BH, et al. Dual role for inflammasome sensors NLRP1 and NLRP3 in murine resistance to Toxoplasma gondii. mBio. 2014;5(1). Epub 2014/02/20. doi: 10.1128/mBio.01117-13. PubMed PMID: 24549849; PubMed Central PMCID: PMCPMC3944820.

23. Dubey JP, Shen SK, Kwok OC, Frenkel JK. Infection and immunity with the RH strain of Toxoplasma gondii in rats and mice. The Journal of Parasitology. 1999;85(4):657-62. PubMed PMID: 10461945.

24. Conrad PA, Miller MA, Kreuder C, James ER, Mazet J, Dabritz H, et al. Transmission of Toxoplasma: clues from the study of sea otters as sentinels of Toxoplasma gondii flow into the marine environment. Int J Parasitol. 2005;35(11-12):1155-68. Epub 2005/09/15. doi: 10.1016/j.ijpara.2005.07.002. PubMed PMID: 16157341.

25. Miller MA, Grigg ME, Kreuder C, James ER, Melli AC, Crosbie PR, et al. An unusual genotype of Toxoplasma gondii is common in California sea otters (Enhydra lutris nereis) and is a cause of mortality. Int J Parasitol. 2004;34(3):275-84. doi: 10.1016/j.ijpara.2003.12.008. PubMed PMID: 15003489.

26. Shapiro K, VanWormer E, Packham A, Dodd E, Conrad PA, Miller M. Type X strains of Toxoplasma gondii are virulent for southern sea otters (Enhydra lutris nereis) and present in felids from nearby watersheds. Proc Biol Sci. 2019;286(1909):20191334. Epub 2019/08/23. doi: 10.1098/rspb.2019.1334. PubMed PMID: 31431162; PubMed Central PMCID: PMCPMC6732395. 
27. Khan A, Dubey JP, Su C, Ajioka JW, Rosenthal BM, Sibley LD. Genetic analyses of atypical Toxoplasma gondii strains reveal a fourth clonal lineage in North America. Int J Parasitol. 2011;41(6):645-55. doi: 10.1016/j.ijpara.2011.01.005. PubMed PMID: 21320505; PubMed Central PMCID: PMC3081397.

28. Dubey JP, Velmurugan GV, Rajendran C, Yabsley MJ, Thomas NJ, Beckmen KB, et al. Genetic characterisation of Toxoplasma gondii in wildlife from North America revealed widespread and high prevalence of the fourth clonal type. Int J Parasitol. 2011;41(11):1139-47. doi: 10.1016/j.ijpara.2011.06.005. PubMed PMID: 21802422.

29. VanWormer E, Miller MA, Conrad PA, Grigg ME, Rejmanek D, Carpenter TE, et al. Using molecular epidemiology to track Toxoplasma gondii from terrestrial carnivores to marine hosts: implications for public health and conservation. PLoS Neglected Tropical Diseases. 2014;8(5):e2852. doi: 10.1371/journal.pntd.0002852. PubMed PMID: 24874796; PubMed Central PMCID: PMC4038486.

30. Gibson AK, Raverty S, Lambourn DM, Huggins J, Magargal SL, Grigg ME. Polyparasitism is associated with increased disease severity in Toxoplasma gondii-infected marine sentinel species. PLoS Neglected Tropical Diseases. 2011;5(5):e1142. doi: 10.1371/journal.pntd.0001142. PubMed PMID: 21629726; PubMed Central PMCID: PMC3101184.

31. Kreuder C, Miller MA, Jessup DA, Lowenstine LJ, Harris MD, Ames JA, et al. Patterns of mortality in southern sea otters (Enhydra lutris nereis) from 1998-2001. J Wildl Dis. 2003;39(3):495-509. doi: 10.7589/0090-3558-39.3.495. PubMed PMID: 14567210.

32. Sundar N, Cole RA, Thomas NJ, Majumdar D, Dubey JP, Su C. Genetic diversity among sea otter isolates of Toxoplasma gondii. Veterinary Parasitology. 2008;151(2-4):125-32. doi: 10.1016/j.vetpar.2007.11.012. PubMed PMID: 18155841.

33. Beraki T, Hu X, Broncel M, Young JC, O'Shaughnessy WJ, Borek D, et al. Divergent kinase regulates membrane ultrastructure of the Toxoplasma parasitophorous vacuole. Proc Natl Acad Sci U S A. 2019;116(13):6361-70. Epub 2019/03/10. doi: 10.1073/pnas.1816161116. PubMed PMID: 30850550; PubMed Central PMCID: PMCPMC6442604.

34. Miller MA, Miller WA, Conrad PA, James ER, Melli AC, Leutenegger CM, et al. Type X Toxoplasma gondii in a wild mussel and terrestrial carnivores from coastal California: new linkages between terrestrial mammals, runoff and toxoplasmosis of sea otters. Int J Parasitol. 2008;38(11):1319-28. doi: 10.1016/j.ijpara.2008.02.005. PubMed PMID: 18452923.

35. Saeij JP, Boyle JP, Boothroyd JC. Differences among the three major strains of Toxoplasma gondii and their specific interactions with the infected host. Trends in Parasitology. 2005;21(10):476-81. doi: 10.1016/j.pt.2005.08.001. PubMed PMID: 16098810.

36. Su C, Khan A, Zhou P, Majumdar D, Ajzenberg D, Darde ML, et al. Globally diverse Toxoplasma gondii isolates comprise six major clades originating from a small number of distinct ancestral lineages. Proc Natl Acad Sci U S A. 2012;109(15):5844-9. doi: 10.1073/pnas.1203190109. PubMed PMID: 22431627; PubMed Central PMCID: PMCPMC3326454.

37. Khan A, Miller N, Roos DS, Dubey JP, Ajzenberg D, Darde ML, et al. A monomorphic haplotype of chromosome Ia is associated with widespread success in clonal and nonclonal populations of Toxoplasma gondii. mBio. 2011;2(6):e00228-11. doi: 
1003

1004

1005

1006

1007

1008

1009

1010

1011

1012

1013

1014

1015

1016

1017

1018

1019

1020

1021

1022

1023

1024

1025

1026

1027

1028

1029

1030

1031

1032

1033

1034

1035

1036

1037

1038

1039

1040

1041

1042

1043

1044

1045

1046

1047

1048

10.1128/mBio.00228-11. PubMed PMID: 22068979; PubMed Central PMCID: PMC3215432.

38. Zhang J, Khan A, Kennard A, Grigg ME, Parkinson J. PopNet: A Markov Clustering Approach to Study Population Genetic Structure. Mol Biol Evol. 2017;34(7):1799-811. Epub 2017/04/07. doi: 10.1093/molbev/msx110. PubMed PMID: 28383661; PubMed Central PMCID: PMCPMC5850731.

39. Sidik SM, Huet D, Ganesan SM, Huynh MH, Wang T, Nasamu AS, et al. A Genome-wide CRISPR Screen in Toxoplasma Identifies Essential Apicomplexan Genes. Cell. 2016;166(6):1423-35 e12. Epub 2016/09/07. doi: 10.1016/j.cell.2016.08.019. PubMed PMID: 27594426; PubMed Central PMCID: PMCPMC5017925.

40. Etheridge RD, Alaganan A, Tang K, Lou HJ, Turk BE, Sibley LD. The Toxoplasma pseudokinase ROP5 forms complexes with ROP18 and ROP17 kinases that synergize to control acute virulence in mice. Cell Host \& Microbe. 2014;15(5):537-50. Epub 2014/05/17. doi: 10.1016/j.chom.2014.04.002. PubMed PMID: 24832449; PubMed Central PMCID: PMCPMC4086214.

41. Khan A, Fux B, Su C, Dubey JP, Darde ML, Ajioka JW, et al. Recent transcontinental sweep of Toxoplasma gondii driven by a single monomorphic chromosome. Proc Natl Acad Sci U S A. 2007;104(37):14872-7. Epub 2007/09/07. doi: 10.1073/pnas.0702356104. PubMed PMID: 17804804; PubMed Central PMCID: PMCPMC1965483.

42. Grigg ME, Sundar N. Sexual recombination punctuated by outbreaks and clonal expansions predicts Toxoplasma gondii population genetics. Int J Parasitol. 2009;39(8):925-33. doi: 10.1016/j.ijpara.2009.02.005. PubMed PMID: 19217909; PubMed Central PMCID: PMC2713429.

43. Ajzenberg D, Cogne N, Paris L, Bessieres MH, Thulliez P, Filisetti D, et al. Genotype of 86 Toxoplasma gondii isolates associated with human congenital toxoplasmosis, and correlation with clinical findings. J Infect Dis. 2002;186(5):684-9. Epub 2002/08/27. doi: 10.1086/342663. PubMed PMID: 12195356.

44. Minot S, Melo MB, Li F, Lu D, Niedelman W, Levine SS, et al. Admixture and recombination among Toxoplasma gondii lineages explain global genome diversity. $\mathrm{P}$ Natl Acad Sci USA. 2012;109(33):13458-63. doi: DOI 10.1073/pnas.1117047109. PubMed PMID: WOS:000307807000067; PubMed Central PMCID: PMCPMC3421188.

45. Khan A, Shaik JS, Behnke M, Wang QL, Dubey JP, Lorenzi HA, et al. NextGen sequencing reveals short double crossovers contribute disproportionately to genetic diversity in Toxoplasma gondii. Bmc Genomics. 2014;15. doi: Artn 1168

10.1186/1471-2164-15-1168. PubMed PMID: WOS:000349049000003.

46. Rajendran C, Su C, Dubey JP. Molecular genotyping of Toxoplasma gondii from Central and South America revealed high diversity within and between populations. Infection, Genetics and Evolution : journal of molecular epidemiology and evolutionary genetics in infectious diseases. 2012;12(2):359-68. Epub 2012/01/10. doi: 10.1016/j.meegid.2011.12.010. PubMed PMID: 22226702.

47. Saeij JP, Boyle JP, Coller S, Taylor S, Sibley LD, Brooke-Powell ET, et al. Polymorphic secreted kinases are key virulence factors in toxoplasmosis. Science. 2006;314(5806):1780-3. doi: 10.1126/science.1133690. PubMed PMID: 17170306; PubMed Central PMCID: PMC2646183.

48. Saeij JP, Coller S, Boyle JP, Jerome ME, White MW, Boothroyd JC. Toxoplasma co-opts host gene expression by injection of a polymorphic kinase homologue. Nature. 
2007;445(7125):324-7. doi: 10.1038/nature05395. PubMed PMID: 17183270; PubMed Central PMCID: PMC2637441.

49. Taylor S, Barragan A, Su C, Fux B, Fentress SJ, Tang K, et al. A secreted serine-threonine kinase determines virulence in the eukaryotic pathogen Toxoplasma gondii. Science. 2006;314(5806):1776-80. doi: 10.1126/science.1133643. PubMed PMID: 17170305.

50. Ferguson D. Toxoplasma gondii and sex: essential or optional extra? Trends in Parasitology. 2002;18(8):351. Epub 2002/10/16. PubMed PMID: 12377284.

51. Fleckenstein MC, Reese ML, Konen-Waisman S, Boothroyd JC, Howard JC, Steinfeldt T. A Toxoplasma gondii pseudokinase inhibits host IRG resistance proteins. PLoS Biology. 2012;10(7):e1001358. doi: 10.1371/journal.pbio.1001358. PubMed PMID: 22802726; PubMed Central PMCID: PMC3393671.

52. Hunn JP, Feng CG, Sher A, Howard JC. The immunity-related GTPases in mammals: a fastevolving cell-autonomous resistance system against intracellular pathogens. Mamm Genome. 2011;22(1-2):43-54. doi: 10.1007/s00335-010-9293-3. PubMed PMID: 21052678; PubMed Central PMCID: PMC3438224.

53. Yamamoto M, Okuyama M, Ma JS, Kimura T, Kamiyama N, Saiga H, et al. A cluster of interferon-gamma-inducible p65 GTPases plays a critical role in host defense against Toxoplasma gondii. Immunity. 2012;37(2):302-13. doi: 10.1016/j.immuni.2012.06.009. PubMed PMID: 22795875.

54. Gazzinelli RT, Mendonca-Neto R, Lilue J, Howard J, Sher A. Innate Resistance against Toxoplasma gondii: An Evolutionary Tale of Mice, Cats, and Men. Cell Host \& Microbe. 2014;15(2):132-8. Epub 2014/02/18. doi: 10.1016/j.chom.2014.01.004. PubMed PMID: 24528860.

55. Koblansky AA, Jankovic D, Oh H, Hieny S, Sungnak W, Mathur R, et al. Recognition of profilin by Toll-like receptor 12 is critical for host resistance to Toxoplasma gondii. Immunity. 2013;38(1):119-30. doi: 10.1016/j.immuni.2012.09.016. PubMed PMID: 23246311; PubMed Central PMCID: PMC3601573.

56. Mackinnon MJ, Read AF. Virulence in malaria: an evolutionary viewpoint. Philosophical transactions of the Royal Society of London Series B, Biological sciences. 2004;359(1446):965-86. doi: 10.1098/rstb.2003.1414. PubMed PMID: 15306410; PubMed Central PMCID: PMC1693375.

57. Ariey F, Witkowski B, Amaratunga C, Beghain J, Langlois AC, Khim N, et al. A molecular marker of artemisinin-resistant Plasmodium falciparum malaria. Nature. 2014;505(7481):50-5. Epub 2013/12/20. doi: 10.1038/nature12876. PubMed PMID: 24352242.

58. Bopp SE, Manary MJ, Bright AT, Johnston GL, Dharia NV, Luna FL, et al. Mitotic evolution of Plasmodium falciparum shows a stable core genome but recombination in antigen families. PLoS Genetics. 2013;9(2):e1003293. doi: 10.1371/journal.pgen.1003293. PubMed PMID: 23408914 ; PubMed Central PMCID: PMC3567157.

59. Miotto O, Almagro-Garcia J, Manske M, Macinnis B, Campino S, Rockett KA, et al. Multiple populations of artemisinin-resistant Plasmodium falciparum in Cambodia. Nature Genetics. 2013;45(6):648-55. Epub 2013/04/30. doi: 10.1038/ng.2624. PubMed PMID: 23624527; PubMed Central PMCID: PMC3807790.

60. Arisue N, Hashimoto T. Phylogeny and evolution of apicoplasts and apicomplexan parasites. Parasitology International. 2014. doi: 10.1016/j.parint.2014.10.005. PubMed PMID: 25451217. 
61. Barbosa L, Johnson CK, Lambourn DM, Gibson AK, Haman KH, Huggins JL, et al. A novel Sarcocystis neurona genotype XIII is associated with severe encephalitis in an unexpectedly broad range of marine mammals from the northeastern Pacific Ocean. Int J Parasitol. 2015. doi: 10.1016/j.ijpara.2015.02.013. PubMed PMID: 25997588.

62. Dubey JP, Graham DH, da Silva DS, Lehmann T, Bahia-Oliveira LM. Toxoplasma gondii isolates of free-ranging chickens from Rio de Janeiro, Brazil: mouse mortality, genotype, and oocyst shedding by cats. The Journal of Parasitology. 2003;89(4):851-3. Epub 2003/10/10. doi: 10.1645/GE-60R. PubMed PMID: 14533703.

63. Wendte JM, Gibson AK, Grigg ME. Population genetics of Toxoplasma gondii: new perspectives from parasite genotypes in wildlife. Veterinary Parasitology. 2011;182(1):96-111. doi: 10.1016/j.vetpar.2011.07.018. PubMed PMID: 21824730.

64. Labruyere E, Lingnau M, Mercier C, Sibley LD. Differential membrane targeting of the secretory proteins GRA4 and GRA6 within the parasitophorous vacuole formed by Toxoplasma gondii. Mol Biochem Parasitol. 1999;102(2):311-24. Epub 1999/09/25. doi: 10.1016/s0166-6851(99)00092-4. PubMed PMID: 10498186.

65. Mercier C, Howe DK, Mordue D, Lingnau M, Sibley LD. Targeted disruption of the GRA2 locus in Toxoplasma gondii decreases acute virulence in mice. Infection and Immunity. 1998;66(9):4176-82. Epub 1998/08/26. PubMed PMID: 9712765.

66. Wang JL, Li TT, Elsheikha HM, Chen K, Zhu WN, Yue DM, et al. Functional Characterization of Rhoptry Kinome in the Virulent Toxoplasma gondii RH Strain. Front Microbiol. 2017;8:84. Epub 2017/02/09. doi: 10.3389/fmicb.2017.00084. PubMed PMID: 28174572; PubMed Central PMCID: PMCPMC5258691.

67. Miller MA, Gardner IA, Packham A, Mazet JK, Hanni KD, Jessup D, et al. Evaluation of an Indirect Fluorescent Antibody Test (Ifat) for Demonstration of Antibodies to Toxoplasma Gondii in the Sea Otter (Enhydra Lutris). Journal of Parasitology. 2002;88(3):594-9. doi: 10.1645/0022-3395(2002)088[0594:eoaifa]2.0.co;2.

68. Pszenny V, Angel So, Duschak VG, Paulino M, Ledesma B, Yabo MI, et al. Molecular cloning, sequencing and expression of a serine proteinase inhibitor gene from Toxoplasma gondii. Mol Biochem Parasitol. 2000;107(2):241-9. PubMed PMID: 10779600.

69. Su C, Zhang X, Dubey JP. Genotyping of Toxoplasma gondii by multilocus PCR-RFLP markers: a high resolution and simple method for identification of parasites. Int J Parasitol. 2006;36(7):841-8. doi: 10.1016/j.ijpara.2006.03.003. PubMed PMID: 16643922.

70. Fazaeli A, Carter PE, Darde ML, Pennington TH. Molecular typing of Toxoplasma gondii strains by GRA6 gene sequence analysis. Int J Parasitol. 2000;30(5):637-42. PubMed PMID: 10779578.

71. Howe DK, Sibley LD. Toxoplasma gondii: analysis of different laboratory stocks of the RH strain reveals genetic heterogeneity. Exp Parasitol. 1994;78(2):242-5. doi: 10.1006/expr.1994.1024. PubMed PMID: 7907030.

72. Gajria B, Bahl A, Brestelli J, Dommer J, Fischer S, Gao X, et al. ToxoDB: an integrated Toxoplasma gondii database resource. Nucleic Acids Res. 2008;36(Database issue):D553-6. doi: 10.1093/nar/gkm981. PubMed PMID: 18003657.

73. Donald RG, Carter D, Ullman B, Roos DS. Insertional tagging, cloning, and expression of the Toxoplasma gondii hypoxanthine-xanthine-guanine phosphoribosyltransferase gene. Use as a selectable marker for stable transformation. J Biol Chem. 
1996;271(24):14010-9. Epub 1996/06/14. doi: 10.1074/jbc.271.24.14010. PubMed PMID: 8662859.

74. Fletcher S. Indirect Fluorescent Antibody Technique in the Serology of Toxoplasma gondii. J Clin Pathol. 1965;18:193-9. Epub 1965/03/01. PubMed PMID: 14276154.

75. Larkin MA, Blackshields G, Brown NP, Chenna R, McGettigan PA, McWilliam H, et al. Clustal W and Clustal $X$ version 2.0. Bioinformatics. 2007;23(21):2947-8. doi: 10.1093/bioinformatics/btm404. PubMed PMID: 17846036.

76. Tamura K, Stecher G, Peterson D, Filipski A, Kumar S. MEGA6: Molecular Evolutionary Genetics Analysis version 6.0. Mol Biol Evol. 2013;30(12):2725-9. doi: 10.1093/molbev/mst197. PubMed PMID: 24132122.

77. Huson DH, Bryant D. Application of phylogenetic networks in evolutionary studies. Mol Biol Evol. 2006;23(2):254-67. doi: 10.1093/molbev/msj030. PubMed PMID: 16221896.

78. Feil EJ, Li BC, Aanensen DM, Hanage WP, Spratt BG. eBURST: Inferring Patterns of Evolutionary Descent among Clusters of Related Bacterial Genotypes from Multilocus Sequence Typing Data. Journal of Bacteriology. 2004;186(5):1518-30. doi: 10.1128/jb.186.5.1518-1530.2004.

79. Rozas J, Sanchez-DelBarrio JC, Messeguer X, Rozas R. DnaSP, DNA polymorphism analyses by the coalescent and other methods. Bioinformatics. 2003;19(18):2496-7. doi: 10.1093/bioinformatics/btg359.

80. Van der Auwera GA, Carneiro MO, Hartl C, Poplin R, Del Angel G, Levy-Moonshine A, et al. From FastQ data to high confidence variant calls: the Genome Analysis Toolkit best practices pipeline. Curr Protoc Bioinformatics. 2013;11(1110):11 0 1- 0 33. doi: 10.1002/0471250953.bi1110s43. PubMed PMID: 25431634.

81. Danecek, P., Auton, A., Abecasis, G., Albers, C. A., Banks, E., DePristo, M. A., Genomes Project Analysis. (2011). The variant call format and VCFtools. Bioinformatics, 27(15), 2156-2158. doi:10.1093/bioinformatics/btr330.

82. Yin, T., Cook, D., \& Lawrence, M. (2012). ggbio: an R package for extending the grammar of graphics for genomic data. Genome Biol, 13(8), R77. doi:10.1186/gb-2012-13-8-r77

83. Behnke, M. S., Khan, A., Lauron, E. J., Jimah, J. R., Wang, Q., Tolia, N. H., \& Sibley, L. D. (2015). Rhoptry Proteins ROP5 and ROP18 Are Major Murine Virulence Factors in Genetically Divergent South American Strains of Toxoplasma gondii. PLoS Genet, 11(8), e1005434. doi:10.1371/journal.pgen.1005434.

84. Smith, R., Sheppard, K., DiPetrillo, K., \& Churchill, G. (2009). Quantitative trait locus analysis using J/qtl. Methods Mol Biol, 573, 175-188. doi:10.1007/978-1-60761-2476_10.

\section{Competing interests}

The authors declare no competing financial interests.

\section{Figure Legends}

\section{Figure 1: Parasite diversity and mouse virulence within the Type $X$ clade}

A) 53 isolates genotyped using 5 unlinked markers identified 9 distinct T. gondii genotypes infecting southern sea otters. Type I, II, and III alleles are represented by red, green, and blue, respectively. Sea otters were infected with T. gondii strains that possessed either a non-archetypal allele (purple or orange) or a Type II allele (green) at the loci 
1187 is shown for each genotype group. Each group was comprised of the following isolates: A $(3097,3142,3168,3265,3483,3488,3520,3637,3659,3744,3786,3821,3865,3947,3950$, 4003, 4045, 4071, 4151), B (3458, 3523, 3728, 3897), C (3026, 3045, 3077, 3160, 4167), D $(3133,3178,3183,3451,4166), \mathbf{E}(3819), \mathbf{F}(3387,3429,3503), \mathbf{G}(3675), \mathbf{H}(3671)$, II (2987, 2994, 3005, 3009, 3087, 3131, 3208, 3396, 3521, 3576, 3587, 3636, 3739, 4181). B) Total Sea Otter Strandings: number of sea otter strandings between 1998-2004 are charted according to their 5 marker type designation of Type II (green) or sub-types of Type $\mathrm{X}$ (range of purples). Type II infections not only clustered geographically (not shown) but also temporally while Type $\mathrm{X}$ infections are steady over time. Additionally, the Type X, subtype A infections which were predominantly avirulent in sea otters show increased sea otter infections over time. representing all singletons $(\mathbf{E}, \mathbf{G}, \mathbf{H})$ and at least two isolates from each of the other 6 distinct genotypes (II, A, B, C, D, F) recovered from sea otters. Mouse seroconversion and survival was monitored for 30 days. At least two independent infection experiments were performed using 5 mice each for every strain tested. Results shown are for those mice that seroconverted or died acutely during infection. Strains are grouped and colored based on their genotype and virulence in mice. Red, mouse virulent, Blue, intermediate mouse virulent (some mice survived acute infection); Green, mouse avirulent (all mice survived acute infection). Number of mice infected by each genotype and their relative virulence is 1207 indicated. D) eBURST analysis to identify linkage disequilibrium across 17 nuclear-encoded linked and unlinked markers identified 4 clonal complexes and 3 unique Type $\mathrm{X}$ genotypes among 21 isolates selected from the 9 distinct clades in Figure 1A. Isolate colors represent the lineage Type from A: red (Type I), green (Type II), blue (Type III), and purple (Type X; all subgroups $(\mathrm{A}-\mathrm{H})$ ). Dot size is proportional to the number of isolates with that genotype; larger dots are multi-isolate genotypes. Clonal complexes (CC) are indicated by lines connecting isolates and are highlighted with ovals corresponding to their allelic identity (Xpurple, II-green)

Figure 2: Phylogenetic analyses support the designation of Type $X$ as a recombinant clade of related strains

1219 Maximum likelihood trees of sequenced markers are depicted. Allele designations were dependent on a bootstrap support greater than 60\%. Isolates are colored based on their genotype from Figure 1A (I: red, II: green, III: blue, X: purple). A) Mitochondrial marker, C01. B) Nuclear genome marker BSR4 on Chromosome IV. At BSR4, all Type X strains possessed a single allele, referred to as $\gamma$ that was readily distinguished from Type I, II and III alleles C) Comparison of two unlinked nuclear-encoded markers GRA7 and BAG1. At both markers, Type X strains possessed one of two distinct alleles, referred to as $\gamma$ or $\delta$. Isolates possessing $\delta$ alleles at both markers were highlighted in purple, those that possessed Type II alleles at both markers were highlighted in green. Isolates that were recombinant and possessed a $\gamma$ allele at GRA7 but a $\delta$ allele at BAG1 were highlighted in pink, and conversely, a $\delta$ allele at GRA7 and a $\gamma$ allele at BAG1 were highlighted in orange. D) Comparison of linked genomic markers GRA7 and SAG4. Genetic recombination is highlighted within this chromosome. 
green. Isolates that show recombination between the $\gamma / \delta$ lineage alleles and the Type II lineage alleles are highlighted in pink and orange depending on recombination directionality.

Figure 3: Genome-wide SNP typing of Type X displays haploblock recombination across the genome

A) CGH-array hybridization of Type X strains against Type I, II and III lineage-specific probes identified 8 distinct Type II and non-Type II hybridization patterns across the 8 Type X strains examined. SNPs are represented by a dot in one of three rows indicating where the isolate has hybridized to the microarray with a hybridization characteristic of lineage Type I (red), II (green), or III (blue). Grey indicates no hybridization at this location. Chromosomes are represented as alternating grey and white bars. B) Linkage disequilibrium and network reticulation demonstrates the existence of recombination across the genomes of Type X. A NeighborNet tree based on whole genome sequence identified 568,592 SNP variant positions across the 19 Type X strains reference mapped to ME49. Type I (red), II (green), III (blue), and X (purple) strains were annotated in the box beside the group based on their previous MLST designation. Two clusters of Type X strains were identified. Strains within the inset were colored based on their murine virulence: virulent (red), intermediate virulent (blue), and avirulent (green).

Figure 4: SNP density plots identify distinct and different recombination crossover points between the Type II and $\gamma / \delta$ lineage among the Type $X$ strains examined WGS reads for all strains were mapped to the ME49 reference genome. Each row represents the SNPs of one strain mapped in a sliding $10 \mathrm{~kb}$ window across the genome, separated into chromosomes columns. Vertical bars across the row represent the number of SNPs, the height of the bar (from y-axis 0-200 bp) represents the amount of divergence from the ME49 genome. Type X strains were grouped based on their MLST designation.

Figure 5: Whole genome sequencing establishes Type $X$ as a recombinant clade of related strains represented by a circle of concatenated chromosomes starting at the top with Chromosome Ia and rotating clockwise to Chromosome XII. All strains were clustered into four distinct color groups based on their degree of shared ancestry (Type II-green, Type X-pink, Type Icyan, Type III-blue) and each isolate has an inner circle that is arrayed in color-group haploblocks based on shared ancestry. Line thickness between strains indicates their interrelatedness, boldest lines indicate $90-100 \%$ of the genome is in linkage disequilibrium, as shown for strains 4167, 3819, 3045 and 3160. is shown in green whereas the mosaic of $\gamma / \delta$ ancestry is shown in black.

C) 21 WGS isolates are shown with their group designation for the 5 loci MLST (Figure 1A), eBURST clonal complex identity (Figure 1D), 17 loci MLST (Table 1), and murine virulence phenotype (Figure 1C). 
1278 A) WGS data from Type X strains was down-selected to one SNP every $5 \mathrm{~kb}$ and analyzed using standard QTL methods with 1000 bootstrap support. LOD scores are shown across the chromosomes for these strains based on genetic association with acute murine virulence as shown in Table 1. B) Significantly associated peaks from the QTL were identified based on clusters of SNP peaks. The LOD scores for the tallest portion of the associated genomic region are listed. C) Distance-based Neighbor-joining ROP33 tree. Alignment of ROP33 DNA sequences were used to construct a phylogenetic tree representing the majority of ROP33 alleles encoded within the species Toxoplasma gondii. Mouse virulence for each isolate is depicted by a colorcode, per Figure 1C. Red, mouse virulent, Blue, intermediate mouse virulent (some CD-1 mice survived acute infection); Green, mouse avirulent (all CD-1 mice survived acute infection). Inoculum size was 50 tachyzoites injected intraperitoneally in CD-1 outbred mice. D) Type I RH parasites deficient in ROP18 and ROP33 are less virulent than Type I RH parasites deficient in ROP18 alone, indicating that ROP33 is a virulence locus in Toxoplasma. CD-1 mice were infected intraperitoneally with 500 tachyzoites of the RH $\Delta$ rop18 or RHArop184rop33 DK0, only the results for seropositive mice are shown. Data are combined from two independent experiments, each with five mice per group. E) Bioluminescent detection of parasite burden in vivo at 9 days post-infection of CD-1 mice with 50 tachyzoites injected intraperitoneally. A representative image with photon output in photons/second/cm2/surface radiance (sr) is shown.

Supplemental Figure 1: Stranding location, necropsy date, sex, Toxoplasma titer (IFAT), and co-infection status of every California Sea otter that yielded a Toxoplasma isolate for genetic characterization

A) Geographical map for all Toxoplasma-infected sea otter stranding locations along the California coastline. Each Sea otter stranding location is represented by a black dot. B) Isolates of Toxoplasma gondii used in this paper. Isolates in yellow were genotyped previously using PCR-RFLP analyses at 4 loci (B1, SAG1, SAG2, SAG3) and isolates 3131, 3133, 3160, 3265 were DNA sequenced at the GRA6 locus [25]. ATOS numbers and stranding locations identify the location of each stranded Sea otter. Isolates were grouped together based on their 5 locus DNA sequence genotype and then by their ATOS number. "Tg" indicates Toxoplasma gondii, "Sn" indicates co-infection with Sarcocystis neurona.

Supplemental Table 1: Expanded sequencing marker genotyping of Type X isolates reveals chromosomal segregation and recombination within chromosomes

21 isolates characterized at 17 nuclear markers (ROP1 was excluded as it is a microsatellite marker, and prone to elevated mutation rates) clade into 12 distinct Type X genotypes (X1$\mathrm{X} 12)$. Isolate numbers in red were sequenced at whole-genome (WGS) resolution. Types I, II, III, $\gamma$ and $\delta$ lineage alleles, as determined by phylogenetic comparisons, are colored red, green, blue, purple, and orange, respectively. Shades of colors represent genetic drift below the $60 \%$ bootstrap delineation from the canonical allele. White represents uninformative or incomplete DNA sequencing results.

Supplemental Table 2: Potential virulence candidate genes derived from the QTL analysis 
1323 Chromosome peaks with LOD scores over 3 as shown in Figure 3B were interrogated via 1324 ToxoDB to select genes in these regions which have predicted signal sequences (Signal) and 1325 transmembrane (TM) domains in Toxoplasma. Genes are listed with gene ID, genomic 1326 location, and predicted protein function. 
bioRxiv preprint doi: https://doi.org/10.1101/2021.03.31.437793; this version posted March 31, 2021. The copyright holder for this preprint (which was not certified by peer review) is the author/funder, who has granted bioRxiv a license to display the preprint in perpetuity. It is made available under aCC-BY-NC-ND 4.0 International license.

\begin{tabular}{|c|c|c|c|c|c|c|c|}
\hline Marker & 胥 & స్ర్ & 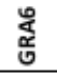 & 몰 & 㨱 & $\begin{array}{l}\text { ○ } \\
\#\end{array}$ & 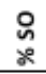 \\
\hline Marker Type & Seq & Seq & Seq & RFLP & Seq & & \\
\hline Chromosome & IV & Vllb & $\mathrm{x}$ & $\mathrm{XI}$ & XII & & \\
\hline Length & 1138 & 1917 & 758 & Dde1 & 253 & & \\
\hline \multicolumn{8}{|l|}{ Type I } \\
\hline \multicolumn{8}{|l|}{ Type II } \\
\hline \multicolumn{8}{|l|}{ Type III } \\
\hline II & & & & & & 14 & 26 \\
\hline A & & 44 & 44 & & & 19 & 36 \\
\hline B & & 44 & $E$ & & & 4 & 7.5 \\
\hline C & & 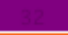 & 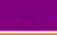 & & & 5 & 9.4 \\
\hline D & & 44 & 44 & & & 5 & 9.4 \\
\hline E & & 32 & 44 & & & 1 & 1.9 \\
\hline $\mathbf{F}$ & & 44 & & & & 3 & 5.7 \\
\hline G & & & & 44 & & 1 & 1.9 \\
\hline $\mathrm{H}$ & & - & & & & 1 & 1.9 \\
\hline
\end{tabular}

\section{B}

Total Sea Otter Deaths

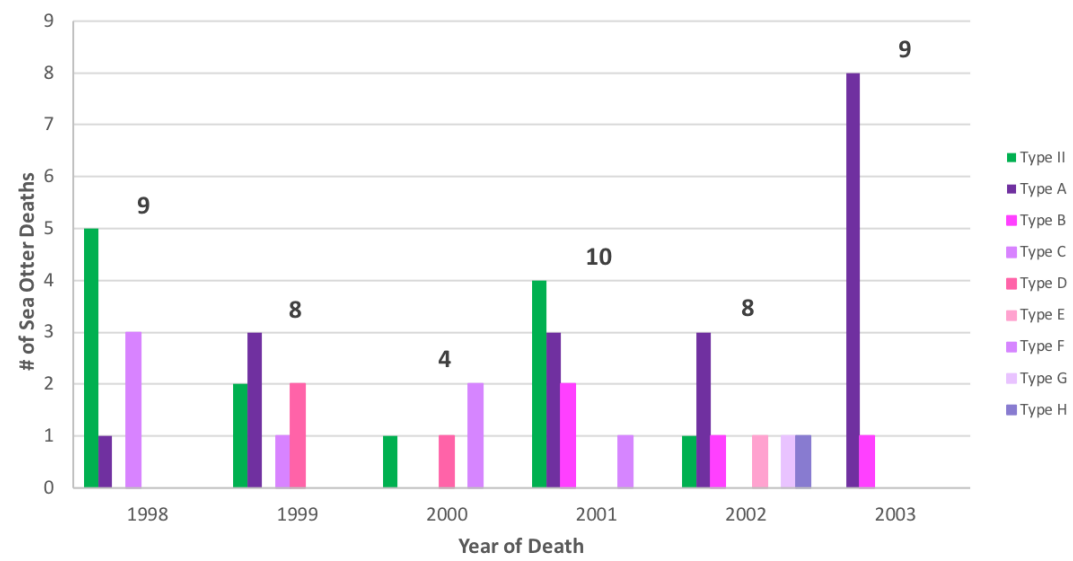

\section{C}

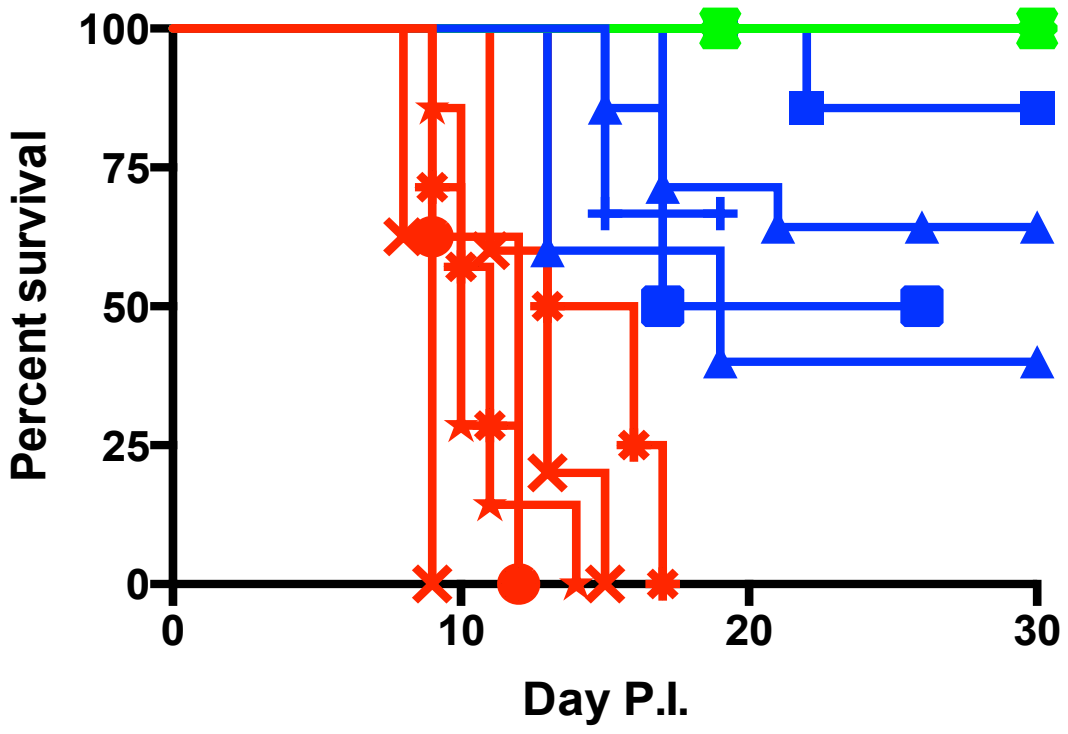

$* A(3142) n=8$

$\star A(3168) n=7$

$-A(3265) n=7$

$+B(3458) n=3$

$\sim B(3523) n=7$

$\rightarrow C(3026) n=7$

ㄷ $\mathrm{C}(3045) \mathrm{n}=8$

다 $C(4167) n=2$

- $D(3133) n=8$

*D (4166) $n=4$

- $E(3819) n=8$

+ $F(3429) n=14$

- $F(3503) n=17$

— $F(3387) n=10$

- G (3675) $n=2$

* H (3671) $n=10$

+ II (2987) $n=3$

- II (3131) $n=2$

D

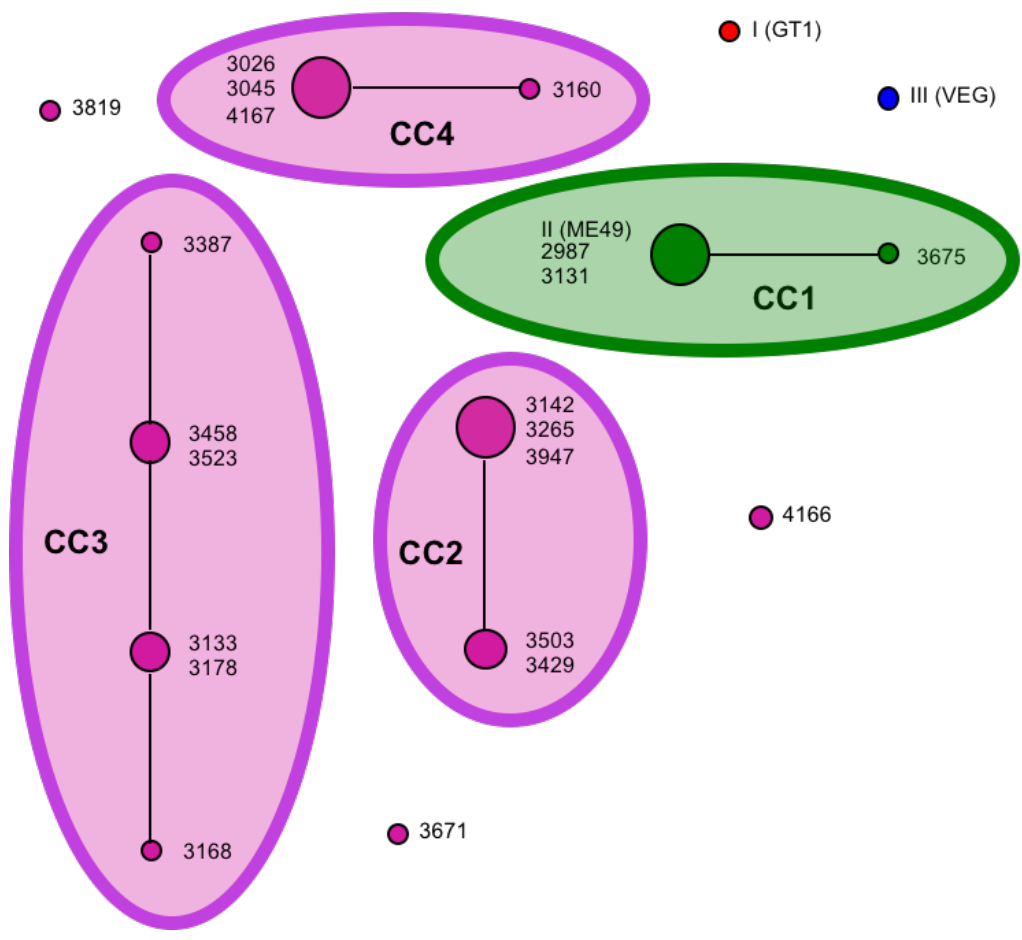


bioRxiv preprint doi: https://doi.org/10.1101/2021.03.31.437793; this version posted March 31, 2021. The copyright holder for this preprint (which was not certified by peer review) is the author/funder, who has granted bioRxiv a license to display the preprint in perpetuity. It is made
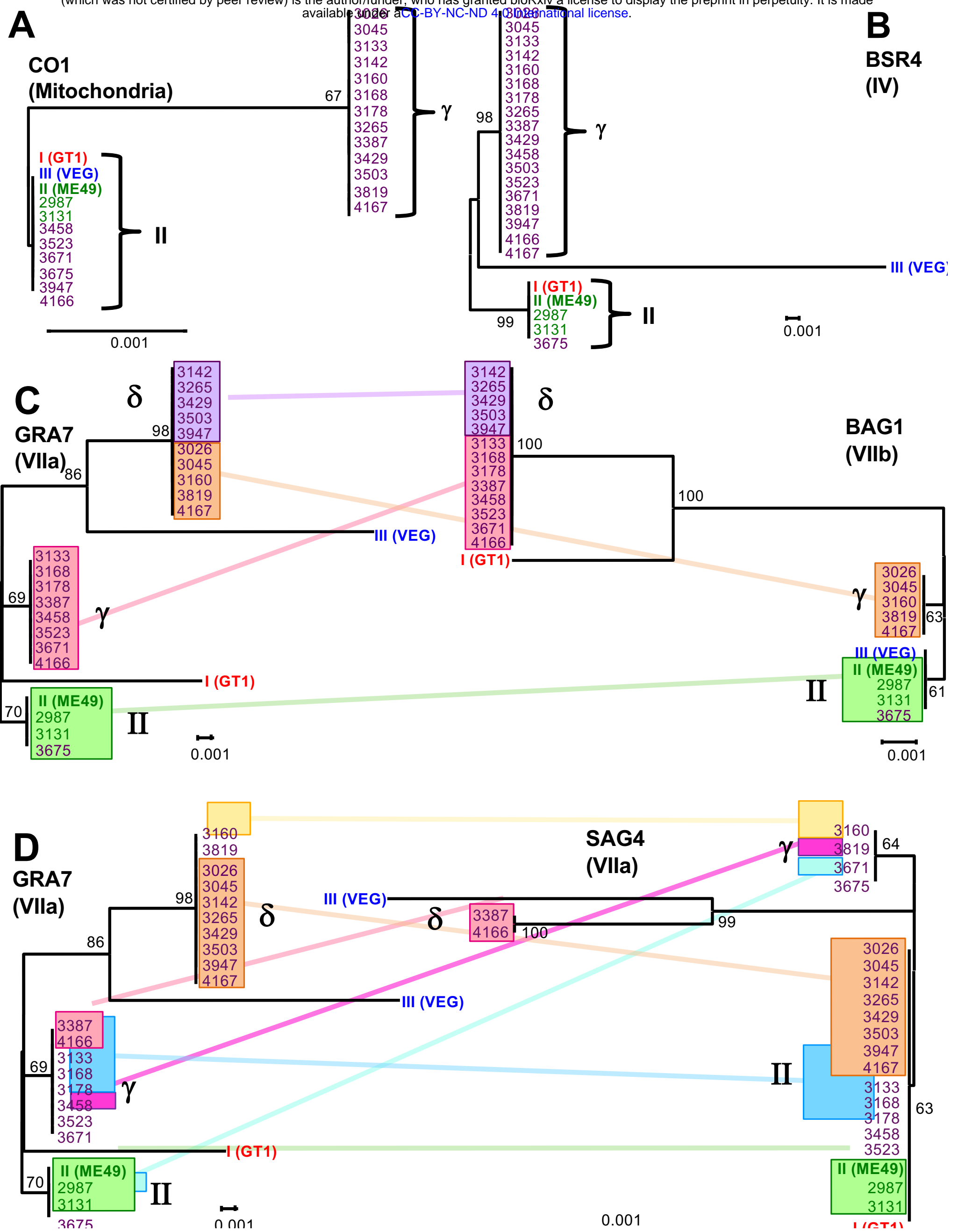
bioRxiv preprint doi: https://doi.org/10.1101/2021.03.31.437793; this version posted March 31, 2021. The copyright holder for this preprint (which was not certified by peer review) is the author/funder, who has granted bioRxiv a license to display the preprint in perpetuity. It is made available under aCC-BY-NC-ND 4.0 International license.

A

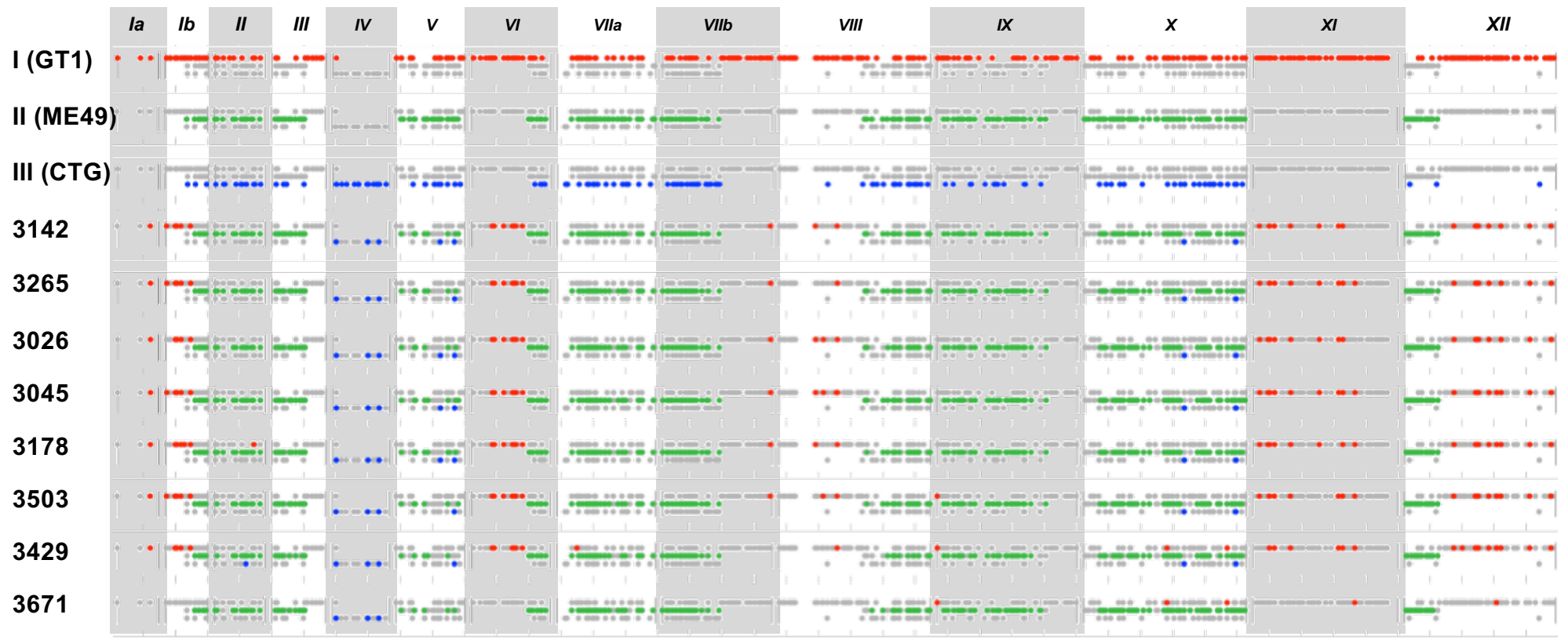

B
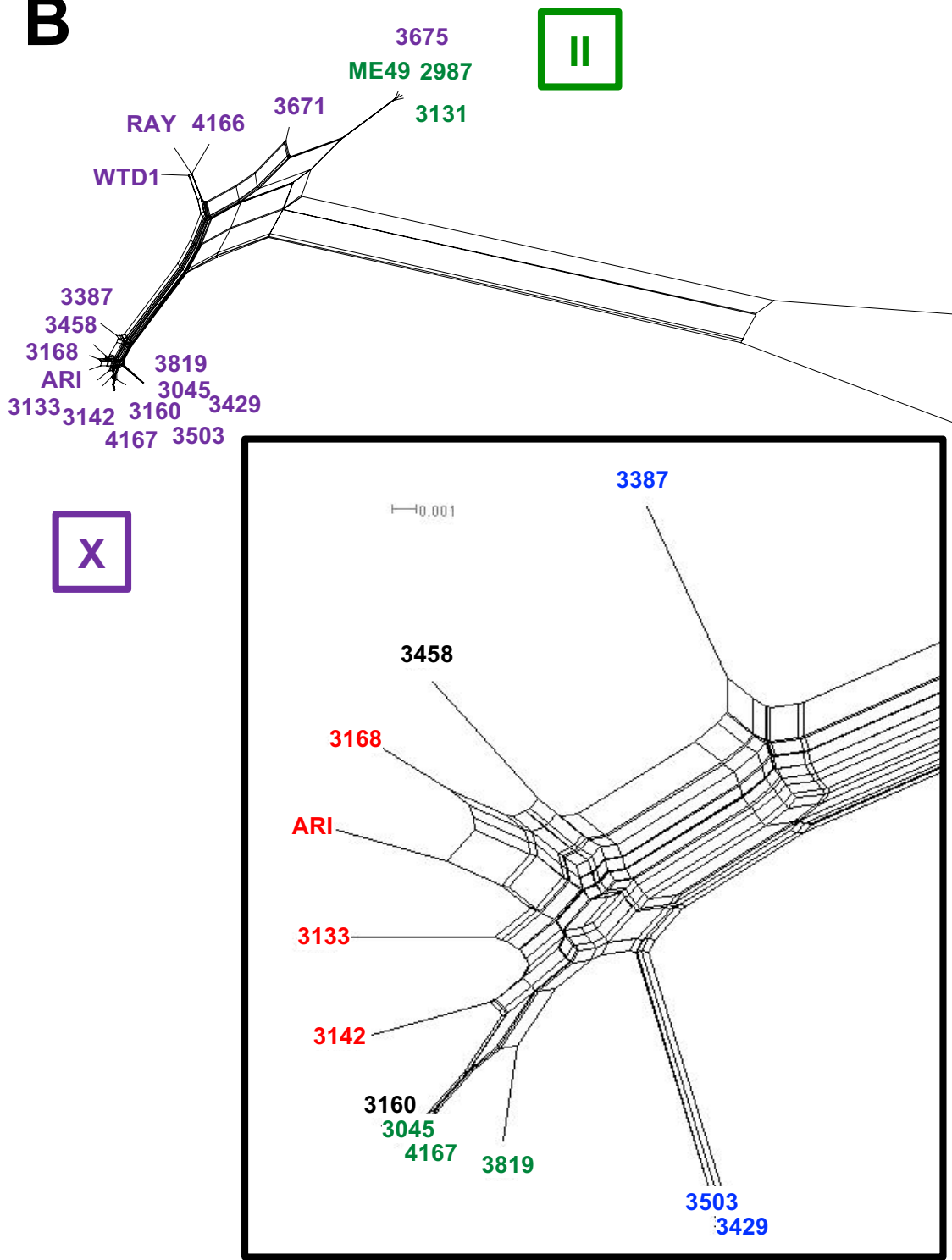

III

VEG 
bioRxiv preprint doi: https://doi.org/10.1101/2021.03.31.437793; this version posted March 31, 2021. The copyright holder for this preprint (which was not certified by peer review) is the author/funder, who has granted bioRxiv a license to display the preprint in perpetuity. It is made available under aCC-BY-NC-ND 4.0 International license.

\section{A}

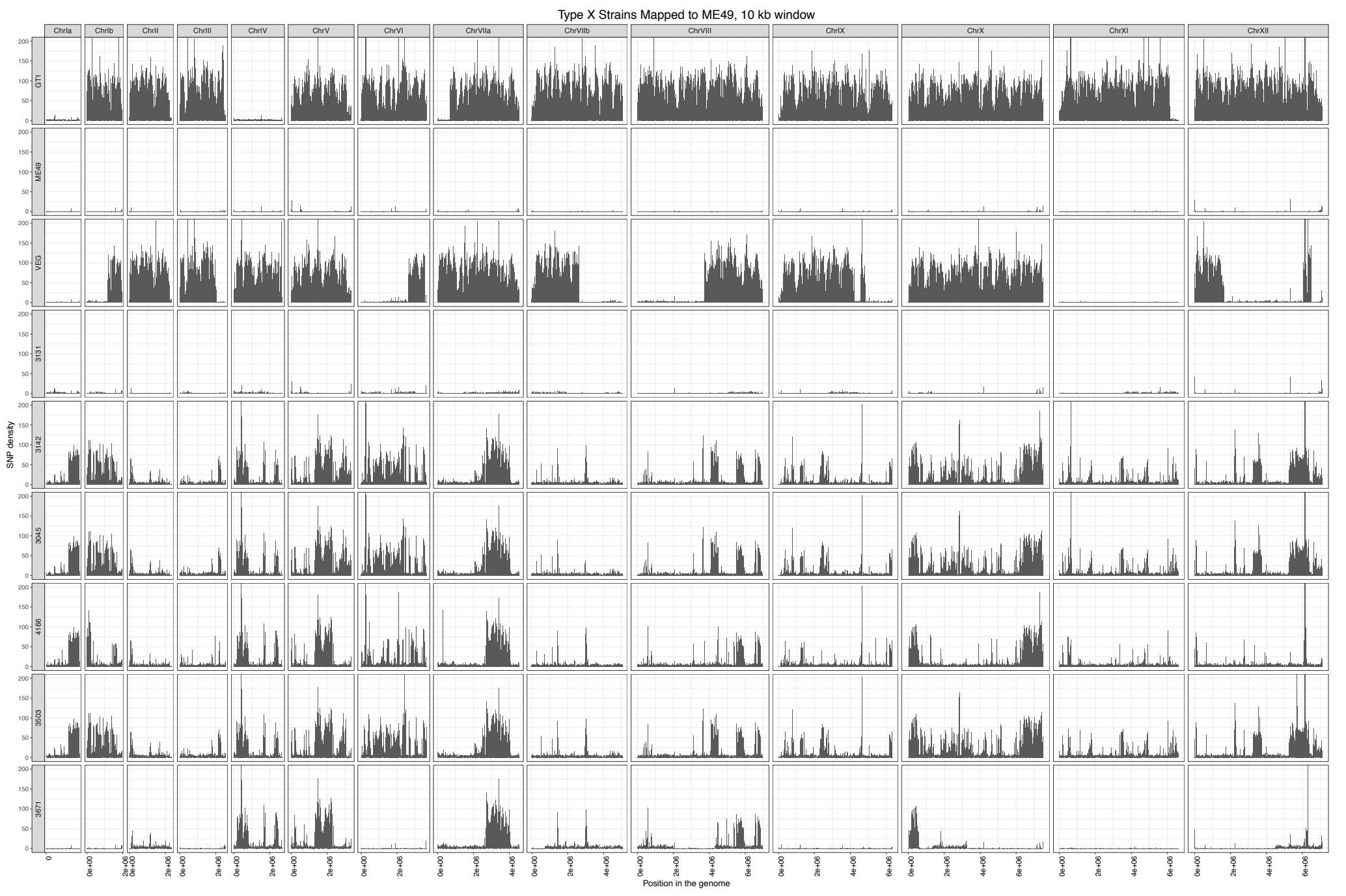


bioRxiv preprint doi: https://doi.org/10.1101/2021.03.31.437793; this version posted March 31, 2021. The copyright holder for this preprint

(which was not certified by peer review) is the author/funder, who has granted bioRxiv a license to display the preprint in perpetuity. It is made

A available under aCC-BY-NC-ND 4.0 International license.

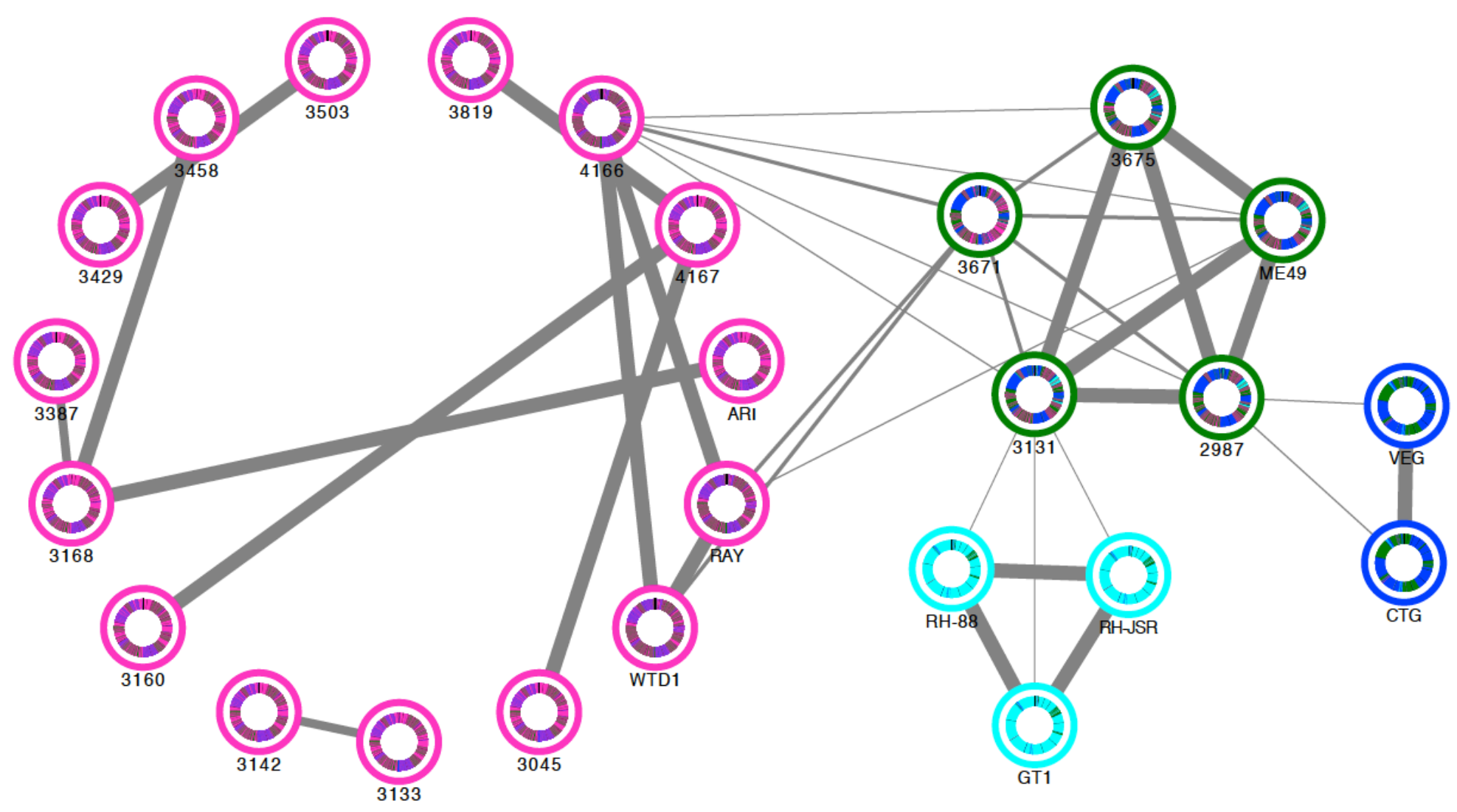

B

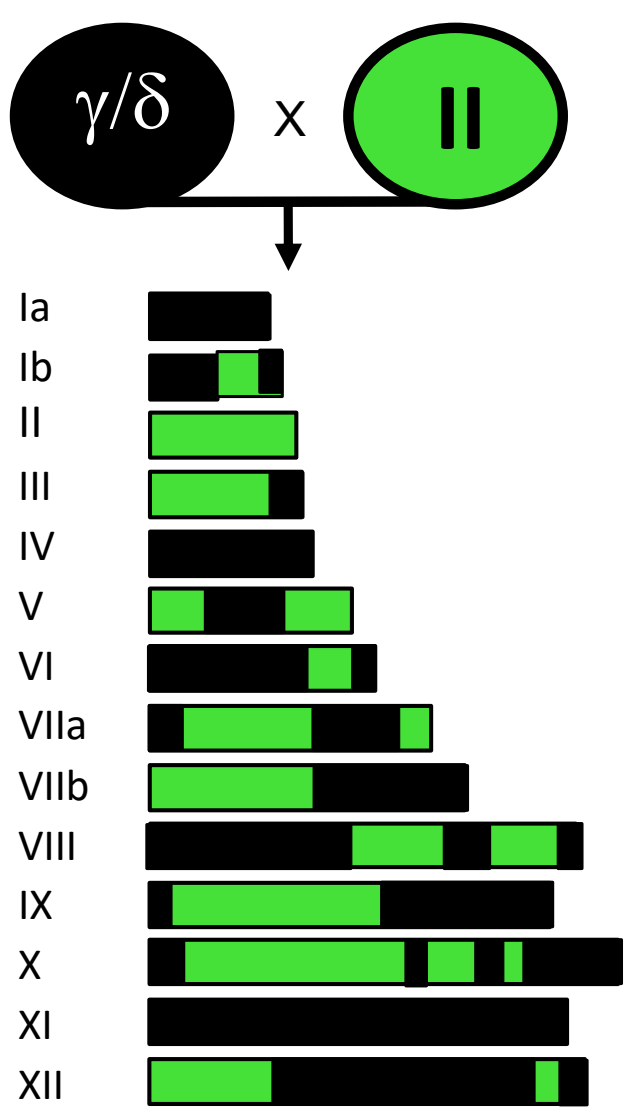

\begin{tabular}{|c|c|c|c|c|}
\hline Isolate & 5 loci & CC & 17 loci & Mouse VIR \\
\hline 2987 & II & CC1 & II & - \\
\hline 3131 & II & CC1 & II & - \\
\hline 3142 & A & CC2 & X1 & + \\
\hline 3265 & A & CC2 & X1 & + \\
\hline 3947 & A & CC2 & X1 & n.d. \\
\hline 3168 & A & CC3 & X2 & + \\
\hline 3458 & B & CC3 & X3 & $+/-$ \\
\hline 3523 & B & CC3 & X3 & - \\
\hline 3026 & C & CC4 & X4 & - \\
\hline 3045 & C & CC4 & X4 & - \\
\hline 4167 & C & CC4 & X4 & $+/-$ \\
\hline 3160 & C & CC4 & X5 & n.d. \\
\hline 3133 & D & CC3 & X6 & + \\
\hline 3178 & D & CC3 & X6 & n.d. \\
\hline 4166 & D & - & X7 & + \\
\hline 3819 & E & - & X8 & - \\
\hline 3503 & F & CC2 & X9 & $+/-$ \\
\hline 3429 & F & CC2 & X9 & $+/-$ \\
\hline 3387 & F & CC3 & X10 & $+/-$ \\
\hline 3675 & G & CC1 & X11 & - \\
\hline 3671 & H & - & X12 & + \\
\hline
\end{tabular}


bioRxiv preprint doi: https://doi.org/10.1101/2021.03.31.437793; this version posted March 31, 2021. The copyright holder for this preprint (which was not certified by peer review) is the author/funder, who has granted bioRxiv a license to display the preprint in perpetuity. It is made

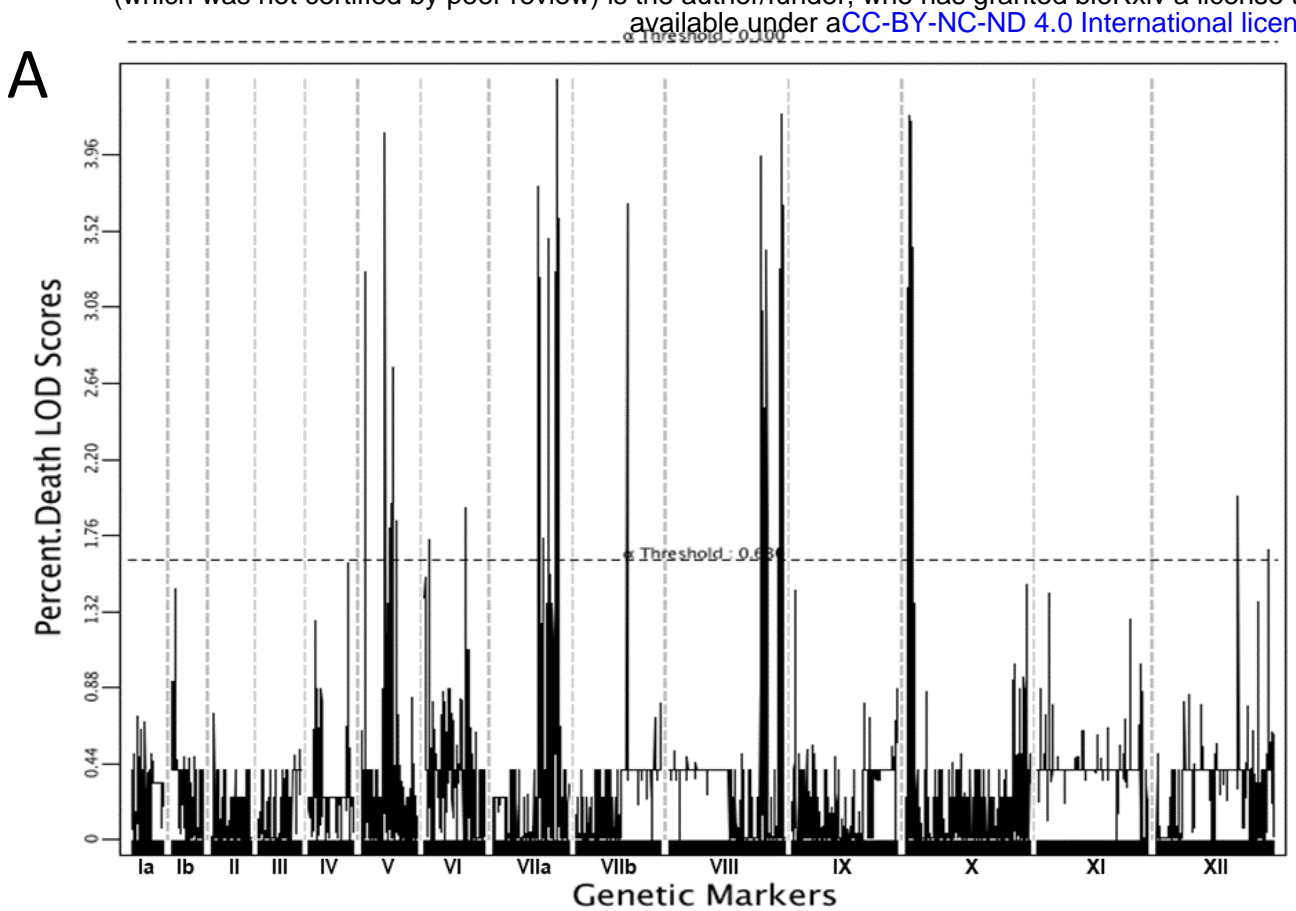

B

\begin{tabular}{|l|l|l|l|}
\hline Chrm & Start (bp) & End (bp) & LOD \\
\hline V & 1430036 & 2165042 & 4.091 \\
\hline VIla & 3815011 & 4085005 & 4.400 \\
\hline VIIb & 3035265 & 3160030 & 3.680 \\
\hline VIII & 6745008 & 6905157 & 4.194 \\
\hline $\mathbf{X}$ & 7314 & 290066 & 4.190 \\
\hline
\end{tabular}
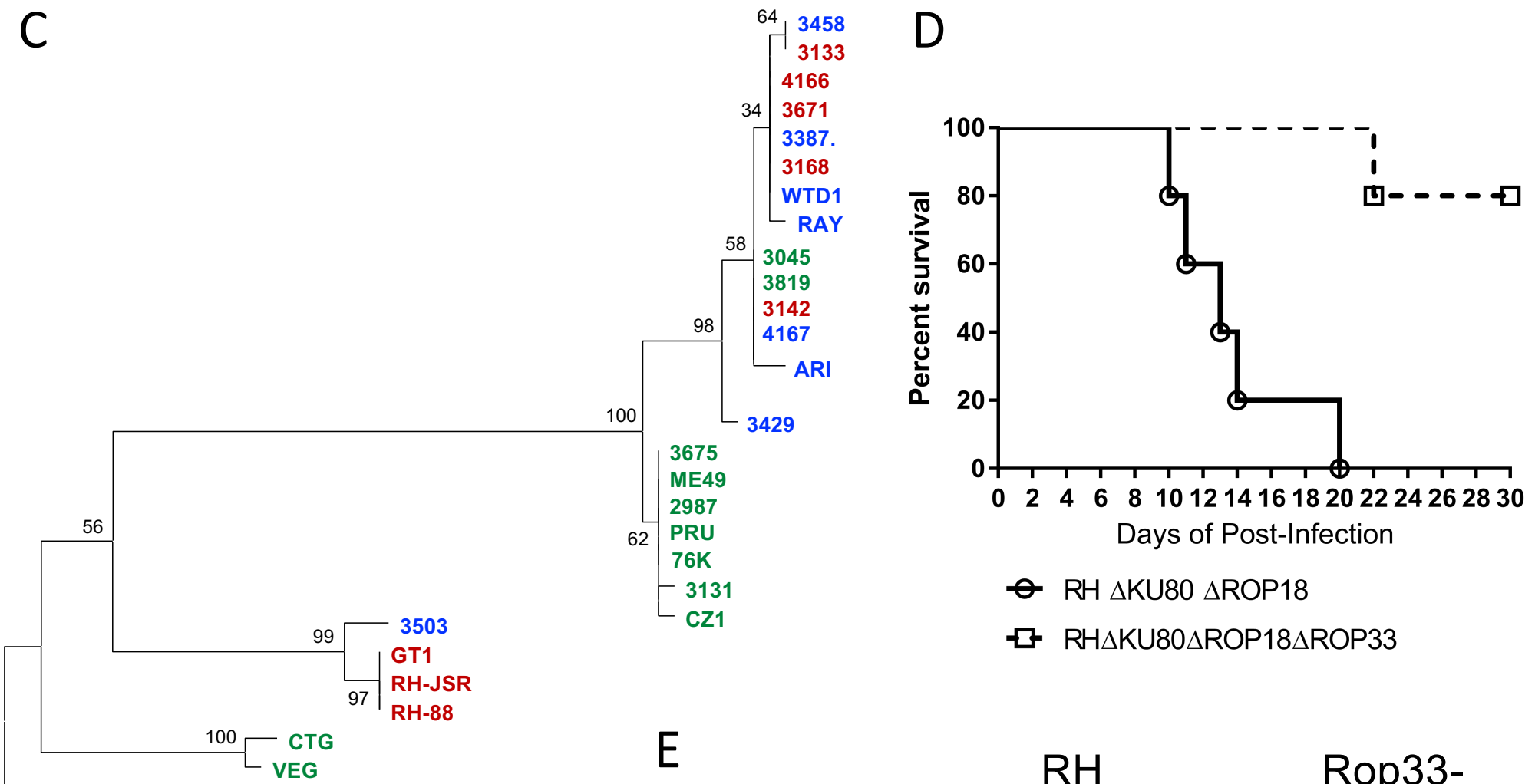

VAND
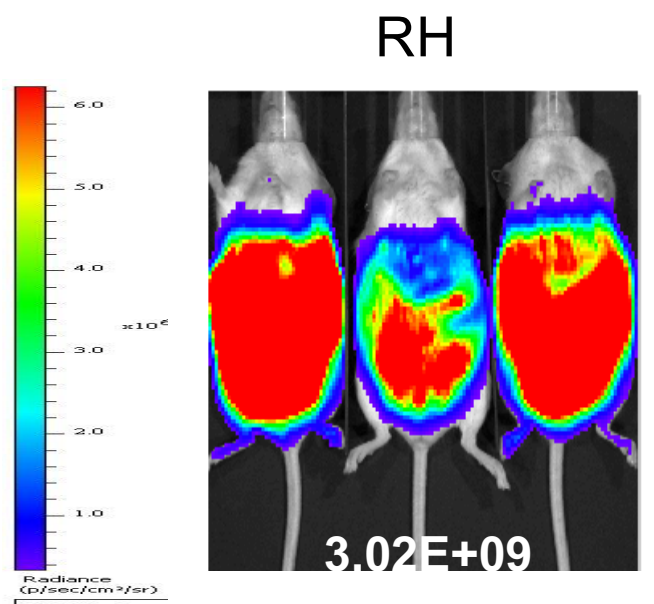

Rop33-

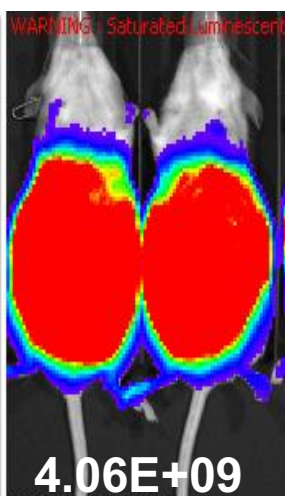


bioRxiv preprint doi: https://doi.org/10.1101/2021.03.31.437793; this version posted March 31, 2021. The copyright holder for this preprint (which was not certified by peer review) is the author/funder, who has granted bioRxiv a license to display the preprint in perpetuity. It is made

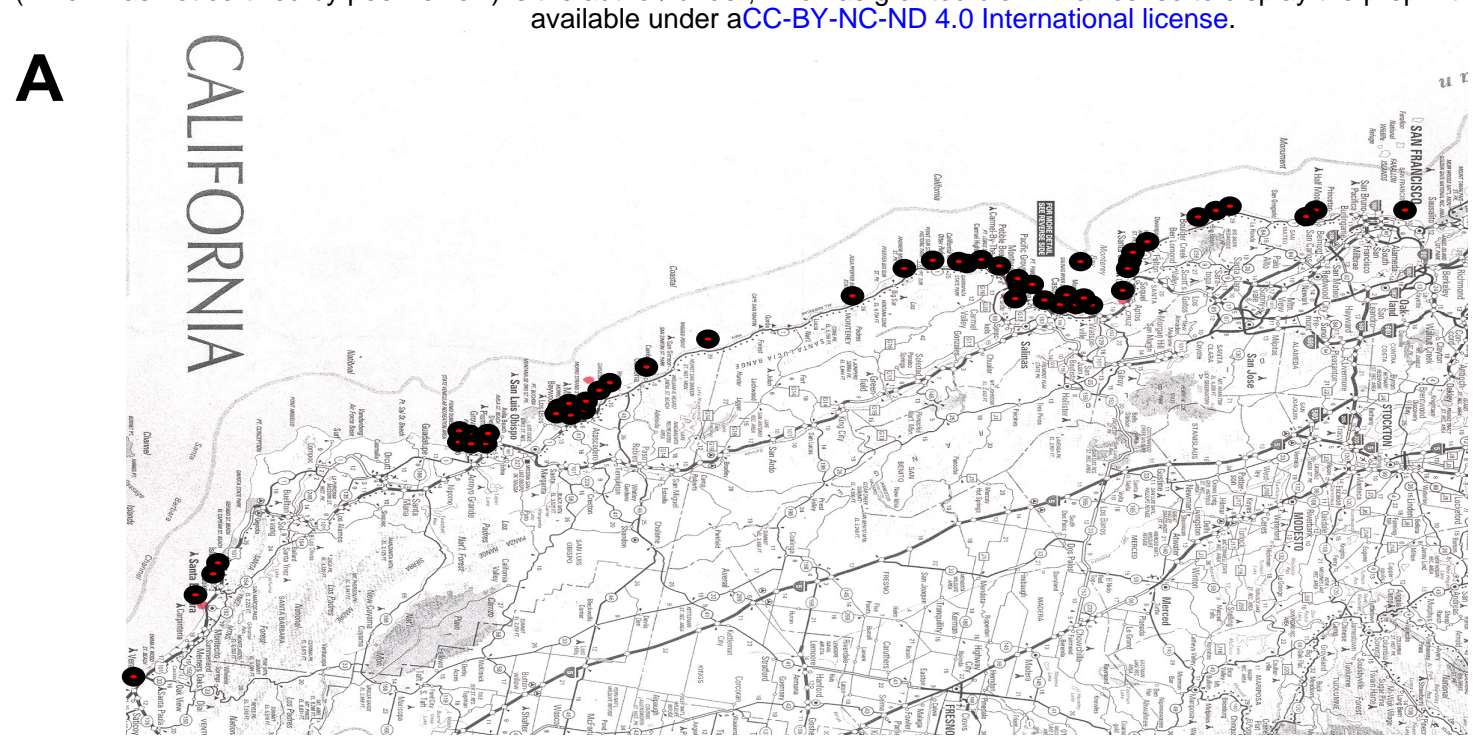

B

\begin{tabular}{|c|c|c|c|c|c|c|c|c|}
\hline Isolate & ATOS \# & 5 loci & 17 loci & Stranding Location & \begin{tabular}{|l|} 
Necropsy \\
Date
\end{tabular} & Sex & IFAT & Infection \\
\hline 2994 & 249 & II & & Santa Cruz & $05 / 15 / 98$ & M & no serum & $\mathrm{Tg}$ \\
\hline 3739 & 264 & II & & Santa Cruz 26th Ave Beach & $06 / 12 / 02$ & M & 2560 & $\mathrm{Tg}$ \\
\hline 3576 & 274 & II & & New Brighton State Beach & 09/05/01 & M & 20480 & $\mathrm{Tg}$ \\
\hline 3587 & 281 & II & & Rio Del Mar Beach & $09 / 22 / 01$ & M & 1280 & $\mathrm{Tg}$ \\
\hline 2987 & 291 & II & III & Mannressa State Beach & $05 / 11 / 98$ & $\mathrm{~F}$ & 1280 & $\mathrm{Tg}$ \\
\hline 4181 & 303 & II & & Sunset State Beach & 04/14/04 & $F$ & 40960 & $\mathrm{Sn} / \mathrm{Tg}$ \\
\hline 3009 & 321 & II & & Elkhorn Slough & $06 / 04 / 98$ & M & 640 & $\mathrm{Tg}$ \\
\hline 3521 & 325 & II & & Salinas River State Beach & $05 / 19 / 01$ & M & 2560 & $\mathrm{Tg}$ \\
\hline 3208 & 357 & II & & Monterey Bay & $07 / 12 / 99$ & M & 5120 & $\mathrm{Tg}$ \\
\hline 3636 & 376 & II & & Del Monte Beach & $12 / 27 / 01$ & M & 80 & $\mathrm{Tg}$ \\
\hline 3087 & 377 & II & & Monterey Harbor & $10 / 28 / 98$ & $F$ & 640 & $\mathrm{Tg}$ \\
\hline 3131 & 379 & II & II & Monterey Bay & $02 / 05 / 99$ & M & 320 & $\mathrm{Tg}$ \\
\hline 3005 & 928 & II & & Pismo Beach & $06 / 01 / 98$ & M & 160 & $\mathrm{Tg}$ \\
\hline 3396 & 928 & II & & Oceano Dunes & $11 / 11 / 00$ & M & 20480 & $\mathrm{Tg}$ \\
\hline 3097 & 84 & A & & Dunes Beach, San Mateo & $11 / 16 / 98$ & M & 5120 & $\mathrm{Tg}$ \\
\hline 3142 & 180 & A & $\mathrm{X} 1$ & Ano Nuevo Island & $02 / 24 / 99$ & $\mathrm{~F}$ & 320 & $\mathrm{Tg}$ \\
\hline 3786 & 180 & A & & Ano Nuevo Island & $09 / 20 / 02$ & M & 2560 & Sn \\
\hline 4045 & 260 & A & & Seabright Beach & $10 / 21 / 03$ & M & 10240 & $\mathrm{Tg}$ \\
\hline 3950 & 263 & A & & Santa Cruz, 21st Street Beach & $07 / 16 / 03$ & M & 10240 & $\mathrm{Sn} / \mathrm{Tg}$ \\
\hline 3520 & 306 & A & & Sunset Beach & $05 / 16 / 01$ & M & 320 & $\mathrm{Tg}$ \\
\hline 3659 & 374 & A & & Del Monte Beach & $02 / 28 / 02$ & $\mathrm{~F}$ & 40 & $\mathrm{Tg}$ \\
\hline 3265 & 426 & A & $\mathrm{X} 1$ & Carmel Beach & $09 / 28 / 99$ & $\mathrm{~F}$ & 10240 & $\mathrm{Tg}$ \\
\hline 3168 & 450 & A & $\mathrm{x} 2$ & Monterey Bay & $04 / 09 / 99$ & $F$ & 2560 & $\mathrm{Tg}$ \\
\hline 3865 & 480 & A & & Carmel River State Beach & $04 / 03 / 03$ & $\mathrm{~F}$ & 10240 & $\mathrm{Tg}$ \\
\hline 3947 & 523 & A & $\mathrm{X} 1$ & Pfieffer Burns Beach & $06 / 26 / 03$ & $\mathrm{~F}$ & 20480 & $\mathrm{Tg}$ \\
\hline 4003 & 750 & A & & Cambria & $09 / 17 / 03$ & $\mathrm{~F}$ & 10240 & $\mathrm{Tg}$ \\
\hline 4071 & 807 & A & & Cayucos, North of E Steet & $12 / 03 / 03$ & $F$ & 2560 & $\mathrm{Tg}$ \\
\hline 3483 & 820 & A & & Morro Strand & $03 / 30 / 01$ & $\mathrm{~F}$ & 320 & $\mathrm{Sn} / \mathrm{Tg}$ \\
\hline 4151 & 825 & A & & Morro Bay & $04 / 04 / 04$ & $\mathrm{~F}$ & $>40960$ & $\mathrm{Sn} / \mathrm{Tg}$ \\
\hline 3488 & 919 & A & & Pismo Beach, $\sim 400 \mathrm{~m}$ North of Grand Ave & $04 / 04 / 01$ & $\mathrm{~F}$ & 640 & $\mathrm{Tg}$ \\
\hline 3821 & 922 & A & & Pismo Beach & $01 / 06 / 03$ & M & 2560 & $\mathrm{Tg}$ \\
\hline 3744 & 924 & A & & Pismo Beach & $07 / 02 / 02$ & $\mathrm{~F}$ & 5120 & $\mathrm{Tg}$ \\
\hline 3637 & 926 & A & & Oceana Dunes & $02 / 14 / 03$ & $\mathrm{~F}$ & no serum & $\mathrm{Tg}$ \\
\hline 3523 & 141 & B & $x 3$ & Pescadero Point & $05 / 31 / 01$ & $\mathrm{~F}$ & 5120 & $\mathrm{Sn} / \mathrm{Tg}$ \\
\hline 3458 & 422 & B & $x 3$ & Stillwater Cove & $01 / 16 / 01$ & $\mathrm{~F}$ & $<80$ & $\mathrm{Tg}$ \\
\hline 3897 & 435 & B & & Pt Lobos State Reserve & $04 / 22 / 03$ & $\mathrm{~F}$ & 10240 & $\mathrm{Tg}$ \\
\hline 3728 & 1,445 & $B$ & & Ventura County (Pt Mugu St Pk) & $05 / 29 / 02$ & M & 320 & $\mathrm{Tg}$ \\
\hline 3077 & 145 & C & & Bean Hollow, Half Moon Bay & $09 / 27 / 98$ & M & 1280 & $\mathrm{Tg}$ \\
\hline 3045 & 808 & C & $\mathrm{X} 4$ & Cayucas & $07 / 09 / 98$ & $\mathrm{~F}$ & no serum & $\mathrm{Tg}$ \\
\hline 3026 & 840 & $\mathrm{C}$ & $\mathrm{X} 4$ & Morro Bay Sandspit & $06 / 24 / 98$ & M & 320 & $\mathrm{Tg}$ \\
\hline 4167 & 852 & $\mathrm{C}$ & $\mathrm{X} 4$ & Morro Creek & $04 / 09 / 04$ & M & 10240 & $\mathrm{Sn} / \mathrm{Tg}$ \\
\hline 3160 & 933 & C & $\mathrm{X} 5$ & Pismo Beach & $03 / 29 / 99$ & M & 320 & $\mathrm{Tg}$ \\
\hline 3178 & 321 & $\mathrm{D}$ & $\mathrm{X} 6$ & Moss Landing Harbor & 03/26/02 & $\mathrm{F}$ & 320 & $\mathrm{Tg}$ \\
\hline 3183 & 816 & $\mathrm{D}$ & & Morro Strand & $05 / 04 / 99$ & $F$ & 640 & $\mathrm{Tg}$ \\
\hline 3133 & 818 & $\mathrm{D}$ & $\mathrm{X} 6$ & Morro Strand Campground & $02 / 04 / 99$ & M & 20480 & $\mathrm{Tg}$ \\
\hline 3451 & 836 & $\mathrm{D}$ & & Morro Bay, Sandspit between port $3 \& 4$ & $11 / 29 / 00$ & M & $<80$ & $\mathrm{Tg}$ \\
\hline 4166 & 827 & $\mathrm{D}$ & $\mathrm{X7}$ & Morro Bay & $04 / 09 / 04$ & M & 40960 & $\mathrm{Sn} / \mathrm{Tg}$ \\
\hline 3819 & 732 & $E$ & $\mathrm{X} 8$ & San Simeon Bay & $12 / 28 / 02$ & $\mathrm{~F}$ & 5120 & $\mathrm{Tg}$ \\
\hline 3429 & 827 & $\mathrm{~F}$ & $\mathrm{X9}$ & Morro Bay & $10 / 30 / 00$ & $\mathrm{~F}$ & 2560 & $\mathrm{Tg}$ \\
\hline 3387 & 922 & $\mathrm{~F}$ & $\mathrm{X} 10$ & Pismo Beach & $07 / 19 / 00$ & $\mathrm{~F}$ & 1280 & $\mathrm{Tg}$ \\
\hline 3503 & 1,319 & $\mathrm{~F}$ & $x 9$ & Rincon Pt., Ventura County & $08 / 09 / 01$ & M & 2560 & $\mathrm{Tg}$ \\
\hline 3675 & 435 & G & $\mathrm{X} 11$ & Point Lobos & $03 / 20 / 02$ & $\mathrm{~F}$ & 1280 & $\mathrm{Tg}$ \\
\hline \multirow[t]{2}{*}{3671} & 321 & $\mathrm{H}$ & $\mathrm{X} 12$ & Elkhorn Slough & $03 / 13 / 02$ & M & 640 & $\mathrm{Sn} / \mathrm{Tg}$ \\
\hline & & $n=53$ & $n=21$ & & & & & \\
\hline
\end{tabular}


bioRxiv preprint doi: https://doi.org/10.1101/2021.03.31.437793; this version posted March 31, 2021. The copyright holder for this preprint

(which was not certified by peer review) is the author/funder, who has granted bioRxiv a license to display the preprint in perpetuity. It is made available under aCC-BY-NC-ND 4.0 International license.
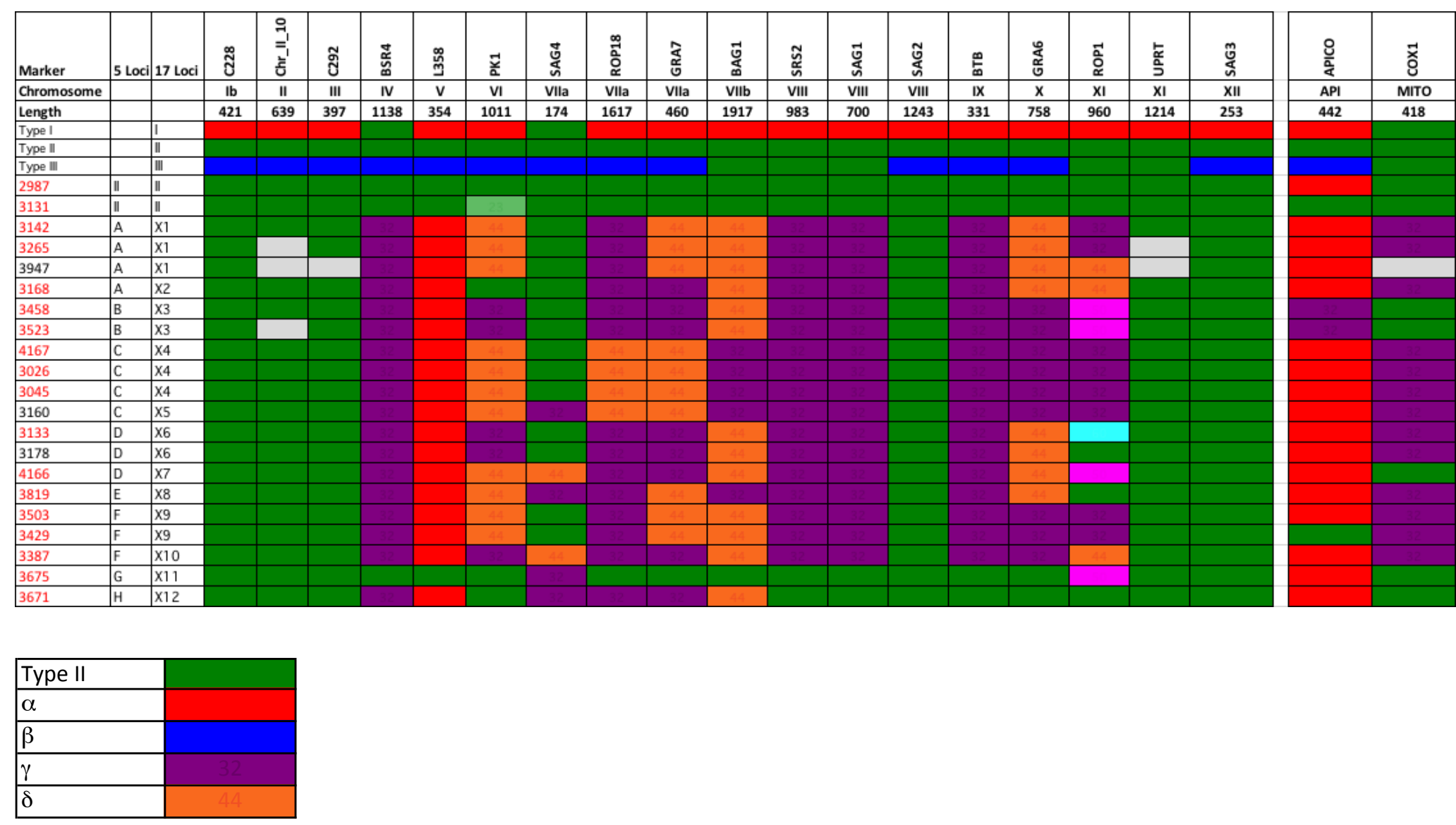
bioRxiv preprint doi: https://doi.org/10.1101/2021.03.31.437793; this version posted March 31, 2021. The copyright holder for this preprint (which was not certified by peer review) is the author/funder, who has granted bioRxiv a license to display the preprint in perpetuity. It is made

\begin{tabular}{|c|c|c|c|c|c|c|c|c|c|}
\hline Chr & Start (bp) & End (bp) & LOD & TM & Signal & Gene ID & valie & $\begin{array}{l}\text { ble under aCe-Bu- } \\
\text { Genomic Location(s) }\end{array}$ & $\begin{array}{l}\text { ipha icicense } \\
\text { Product Description }\end{array}$ \\
\hline \multirow[t]{4}{*}{5} & 220001 & 350021 & 3.25 & Y & Y & TGME49 & 220240 & TGME49_chrV:235,019..240,463(-) & hypothetical protein \\
\hline & & & & Y & Y & TGME49, & 220300 & TGME49_chrV:275,453..283,005(-) & ribosomal protein L15 protein \\
\hline & & & & Y & Y & TGME49 & 220330 & TGME49_chrV:295,153..299,369(-) & hypothetical protein \\
\hline & & & & Y & Y & TGME49, & 220350 & TGME49_chrV:310,715..318,211(+) & tRNA ligases class II ( $\mathrm{D}, \mathrm{K}$ and $\mathrm{N})$ domain-containing protein \\
\hline \multirow[t]{9}{*}{5} & 1430036 & 2165042 & 4.09 & Y & Y & TGME49 & 285870 & TGME49_chrV:2,158,544..2,162,541(-) & SAG-related sequence SRS2OA \\
\hline & & & & Y & Y & TGME49. & 285940 & TGME49_chrV:2,123,872..2,135,543(-) & hypothetical protein \\
\hline & & & & $\mathrm{Y}$ & Y & TGME49 & 286180 & TGME49_chrV:1,933,873..1,942,288(-) & tRNA ligases class I (M) protein \\
\hline & & & & Y & Y & TGME49 & 286450 & TGME49_chrV:1,824,587..1,826,598(+) & dense granule protein GRA5 \\
\hline & & & & $\mathrm{Y}$ & Y & TGME49, & 286530 & TGME49_chrV:1,790,919..1,793,716(+) & hypothetical protein \\
\hline & & & & Y & Y & TGME49 & 286620 & TGME49_chrV:1,754,511..1,762,820(-) & S1 RNA binding domain-containing protein \\
\hline & & & & Y & Y & TGME49, & 286630 & TGME49_chrV:1,750,888..1,752,970(+) & redoxin domain-containing protein \\
\hline & & & & Y & Y & TGME49, & 286770 & TGME49_chrV:1,682,413..1,683,569(+) & hypothetical protein \\
\hline & & & & Y & Y & TGME49 & 213920 & TGME49_chrV:1,428,849..1,440,483(-) & hypothetical protein \\
\hline \multirow[t]{5}{*}{$7 a$} & 3815011 & 4085005 & 4.40 & Y & Y & TGME49 & 201130 & TGME49_chrVIla:4,019,644..4,026,659(+) & rhoptry kinase family protein ROP33 \\
\hline & & & & Y & Y & TGME49 & 201180 & TGME49_chrVIla:3,982,746..3,988,716(-) & hypothetical protein \\
\hline & & & & Y & Y & TGME49, & 201390 & TGME49_chrVIla:3,908,769..3,911,287(-) & hypothetical protein \\
\hline & & & & Y & Y & TGME49 & 201750 & TGME49_chrVIlla: 3,822,835 - 3,823,206(+) & Tctex-1 family protein \\
\hline & & & & Y & Y & TGME49, & 201760 & TGME49_chrVIla: 3,816,484 - 3,820,369(+) & hypothetical protein \\
\hline \multirow[t]{8}{*}{$7 \mathrm{~b}$} & 3035265 & 3160030 & 3.68 & Y & Y & TGME49, & 258828 & TGME49_chrVIIb:3,159,970..3,160,907(+) & hypothetical protein \\
\hline & & & & Y & Y & TGME49, & 258840 & TGME49_chrVIIb:3,137,682..3,141,698(+) & hypothetical protein \\
\hline & & & & Y & Y & TGME49, & 258860 & TGME49_chrVIIb:3,122,191..3,126,503(+) & hypothetical protein \\
\hline & & & & Y & Y & TGME49 & 258890 & TGME49_chrVIIlb:3,103,220..3,104,003(-) & hypothetical protein \\
\hline & & & & Y & Y & TGME49 & 258910 & TGME49_chrVIIb:3,092,653..3,098,081(+) & hypothetical protein \\
\hline & & & & Y & Y & TGME49. & 258950 & TGME49_chrVIIb:3,079,180..3,083,732(+) & lectin family protein \\
\hline & & & & Y & Y & TGME49 & 258990 & TGME49_chrVIIb:3,038,025..3,049,513(+) & Bromodomain-containing protein \\
\hline & & & & Y & Y & TGME49. & 259000 & TGME49_chrVIIb:3,035,953..3,049,513(+) & hypothetical protein \\
\hline \multirow[t]{9}{*}{8} & 5435051 & 6125010 & 3.8 & Y & Y & TGME49 & 269150 & TGME49_chrVIII:6,039,099..6,042,614(-) & DHHC zinc finger domain-containing protein \\
\hline & & & & Y & Y & TGME49, & 269190 & TGME49_chrVIII:6,002,854..6,007,557(-) & glyceraldehyde-3-phosphate dehydrogenase GAPDH2 \\
\hline & & & & Y & Y & TGME49 & 269400 & TGME49_chrVIII:5,836,645..5,840,235(+) & oxidoreductase, short chain dehydrogenase/reductase family protein \\
\hline & & & & Y & Y & TGME49. & 269750 & TGME49_chrVIII:5,625,999..5,628,567(+) & CrcB family protein \\
\hline & & & & Y & Y & TGME49, & 269885 & TGME49_chrVIII:5,564,529..5,572,853(+) & rhoptry metalloprotease toxolysin TLN1 \\
\hline & & & & Y & Y & TGME49, & 269920 & TGME49_chrVIII:5,535,689..5,545,827(-) & phosphatidylserine decarboxylase \\
\hline & & & & Y & Y & TGME49 & 269980 & TGME49_chrVIII:5,483,536..5,489,390(-) & preprotein translocase Sec 61 , putative \\
\hline & & & & Y & Y & TGME49, & 269690 & TGME49_chrVIII:5,680,572..5,684,829(+) & hypothetical protein \\
\hline & & & & Y & Y & TGME49 & 270030 & TGME49_chrVIII:5,448,751..5,449,486(+) & hypothetical protein \\
\hline \multirow[t]{2}{*}{8} & 6745008 & 6905157 & 4.19 & Y & Y & TGME49, & 200230 & TGME49_chrVIII:6,753,611..6,755,422(-) & microneme protein MIC17C \\
\hline & & & & Y & Y & TGME49 & 200440 & TGME49_chrVIII:6,883,172..6,887,049(+) & hypothetical protein \\
\hline 10 & 280002 & 290066 & 4.19 & N/A & N/A & N/A & & N/A & N/A \\
\hline
\end{tabular}

